\title{
EFFECT OF HYDROGEN ON THE TENSILE PROPERTIES OF 42CrMO4 STEEL QUENCHED AND TEMPERED AT DIFFERENT TEMPERATURES
}

\author{
A. Zafra ${ }^{1 *}$, L.B. Peral*, J. Belzunce* and C. Rodríguez* \\ ${ }^{1}$ Corresponding author: Alfredo Zafra García (+34 985182023) alfredyzafra@ hotmail.com \\ *University of Oviedo, campus universitario, east building, 33203, Gijón, Spain
}

\begin{abstract}
The influence of hydrogen on the mechanical behaviour of a 42CrMo4 tempered martensitic steel was investigated by means of tensile tests on both smooth and circumferentially-notched round-bar specimens pre-charged with gaseous hydrogen in a pressurized reactor.

Hydrogen solubility was seen to decrease with increasing tempering temperature. Moreover, hydrogen embrittlement measured in notched specimens was much greater in the grades with higher hardness, tempered at the lowest temperatures, where a change in the fracture micromechanism from ductile in the absence of hydrogen to intermediate and brittle in the presence of hydrogen was clearly observed. Results were discussed through FEM simulations of local stresses acting on the process zone.
\end{abstract}

KEYWORDS: Hydrogen embrittlement, structural steels, notched tensile strength, failure micromechanisms.

\section{INTRODUCTION}

The global trend to replace fossil fuels with cleaner and renewable alternatives is boosting the development of hydrogen-based energy sources. In this context, the expected increase in the demand for hydrogen in the coming years means that current storage and delivering facilities and materials will need to evolve so as to be able to deal with larger amounts of hydrogen in an efficient way. Furthermore, to fulfil this demand, transport and storage units (pipes and vessels) will be required to withstand higher hydrogen pressures, of up to $70 \mathrm{MPa}$.

Hydrogen systems have typically been designed to employ metallic materials with a high resistance to hydrogen embrittlement, such as austenitic stainless steel and aluminium alloys. However, these metals have lower strength and are more expensive than conventional structural steels, such as carbon and lowalloy steels [1]. The economical solution would be to use medium- and high-strength tempered martensitic steels, which allow decreasing pipe and vessel thicknesses and hence the cost of the steel.

$42 \mathrm{CrMo} 4$ is a medium carbon Cr-Mo alloyed steel that is frequently used in a quenched and tempered condition when a good combination of strength and toughness is required. It also has high fatigue strength and wear resistance, making it an excellent candidate for manufacturing vessels working under highpressure hydrogen gas [2]. Nevertheless, it is well known that these steel grades are more sensitive to hydrogen embrittlement (HE) than low-strength steels, this susceptibility increasing with the strength level of the steel [3,4]. As a result, $42 \mathrm{CrMo} 4$ steel is usually tempered at high temperatures with reduced strength and hardness so as to reduce HE-related problems.

Although the deleterious effects of hydrogen on steels similar to $42 \mathrm{CrMo} 4$ have been studied for some time now, the mechanism of hydrogen embrittlement phenomena is still controversial. Anyway, the common understanding is that mechanical loads, hydrogen uptake, diffusion of mobile hydrogen and, of course, the steel microstructure are involved $[4,5,6]$. The mechanism of hydrogen-induced failure in quenched and 
tempered steels usually starts with the stress activation of slip systems in individual grains, being known that the enhancement of this dislocation activity is due to the hydrogen-enhanced localized plasticity (HELP) mechanism [7, 8]. Hydrogen trapping by dislocations reduces the elastic energy between them and thereby decreases the Peierls stress. As a result the mobility of dislocations increases and plasticity localizes [9]. In the case of high strength steels, their final failure can be intergranular, promoted by carbide precipitation and solute content along austenitic grain boundaries, or transgranular associated with cracking along martensitic block and packet interfaces. Moreover, despite many studies about mobile and trapped hydrogen, it is difficult to establish a consensus on the impact of both hydrogen states on damage mechanisms. In general, segregation of hydrogen is necessary to reduce the interface energy and induce a cracking process, although it is argued that the mobile hydrogen reduces the cohesion energy greater than trapped hydrogen [10].

On the other hand, the effect of hydrogen on the mechanical behaviour of high strength steels may depend on the dimensions and geometry of the specimen, on the stress concentration factor, when notched specimens are employed, as well as on certain test variables, such as the strain rate [4]. It is also important to note that embrittlement evaluation is often conducted on mechanical tests performed in samples that were electrochemically charged with hydrogen, but it is known that electrochemical charging produce high hydrogen fugacity near the surface and sometimes causes surface cracking or blistering, so these type of tests should be considered with caution when materials are exposed to gaseous hydrogen [11].

In this context, further investigation is also needed for steels of this kind heat treated at intermediate and high strength levels, as these grades are more susceptible to hydrogen embrittlement. In fact, the main objective of this study is to analyse the influence of heat treatment (specifically the influence of tempering temperature) on the hydrogen embrittlement susceptibility of $42 \mathrm{CrMo} 4$ steel. Hence, the tensile behaviour of both smooth and circumferentially-notched round-bar specimens pre-charged with gaseous hydrogen was compared. Furthermore, different displacement rates were used to analyse the influence of the strain rate on hydrogen embrittlement. FEM simulations of the notched tensile tests were performed and along with the observed fracture micromechanisms, a discussion of failure processes in the presence of hydrogen was provided.

\section{EXPERIMENTAL PROCEDURE}

\subsection{Materials and heat treatments}

A $42 \mathrm{CrMo} 4$ steel was used in the present study. Its chemical composition in weight $\%$ is shown in Table 1.

\begin{tabular}{|c|c|c|c|c|c|c|c|}
\hline Steel & $\mathrm{C}$ & $\mathrm{Mn}$ & $\mathrm{Si}$ & $\mathrm{P}$ & $\mathrm{S}$ & $\mathrm{Cr}$ & Mo \\
\hline 42CrMo4 & 0.42 & 0.62 & 0.18 & 0.008 & 0.002 & 0.98 & 0.22 \\
\hline
\end{tabular}

Table 1. Chemical composition in weight\% of $42 \mathrm{CrMo} 4$ steel.

Hot rolled plates $\left(250 \times 250 \times 12 \mathrm{~mm}^{3}\right)$ of $42 \mathrm{CrMo} 4$ were austenitized at $845^{\circ} \mathrm{C}$ for $40 \mathrm{~min}$, quenched in water, and then tempered at different temperatures, 500, 550, 600, 650 and $700^{\circ} \mathrm{C}$, for 2 hours. The reason for using different heat treatments was to obtain different steel grades in order to analyse the influence of microstructure and strength level on the susceptibility of the steel to hydrogen embrittlement. The sequence of heat treatments and the nomenclature of the $42 \mathrm{CrMo} 4$ steel grades are shown in Table 2.

\begin{tabular}{|c|c|}
\hline Specimen ID & Heat treatments \\
\hline 42CrMo4_700 & $845^{\circ} \mathrm{C} / 40 \mathrm{~min}+$ water quenching $+700^{\circ} \mathrm{C} / 2 \mathrm{~h}$ tempering \\
\hline 42CrMo4_650 & $845^{\circ} \mathrm{C} / 40 \mathrm{~min}+$ water quenching $+650^{\circ} \mathrm{C} / 2 \mathrm{~h}$ tempering \\
\hline 42CrMo4_600 & $845^{\circ} \mathrm{C} / 40 \mathrm{~min}+$ water quenching $+600^{\circ} \mathrm{C} / 2 \mathrm{~h}$ tempering \\
\hline 42CrMo4_550 & $845^{\circ} \mathrm{C} / 40 \mathrm{~min}+$ water quenching $+550^{\circ} \mathrm{C} / 2 \mathrm{~h}$ tempering \\
\hline 42CrMo4_500 & $845^{\circ} \mathrm{C} / 40 \mathrm{~min}+$ water quenching $+500^{\circ} \mathrm{C} / 2 \mathrm{~h}$ tempering \\
\hline
\end{tabular}

Table 2. Heat treatments applied to the $42 \mathrm{CrMo} 4$ steel.

\subsection{Hydrogen charging}


All the specimens were pre-charged with gaseous hydrogen in a high-pressure reactor (with a diameter of $73 \mathrm{~mm}$ and total length of $180 \mathrm{~mm}$ ), manufactured in accordance with the ASTM G146 standard [12]. Before hydrogen charging, air was removed from the vessel using three alternate inert gas purges to reduce the oxygen level to a minimum. To ensure that the specimens were saturated with hydrogen, they were kept in contact with gaseous hydrogen under a pressure of $19.5 \mathrm{MPa}$ at $450^{\circ} \mathrm{C}$ for $21 \mathrm{~h}$. After this holding time, the reactor was cooled down for $1 \mathrm{~h}$ until reaching a temperature of $85^{\circ} \mathrm{C}$. During this phase, the hydrogen pressure was maintained constant at $19.5 \mathrm{MPa}$ to reduce hydrogen egress but, nevertheless, significant hydrogen loss took always place. All the pre-charged specimens were subsequently removed from the reactor and immersed in liquid nitrogen $\left(-196^{\circ} \mathrm{C}\right)$, where they were kept until the start of the corresponding mechanical test, once again in order to limit hydrogen losses.

\subsection{Measurement of hydrogen content}

The hydrogen desorption curves of the different steel grades at room temperature were determined. These curves represent the hydrogen content versus time ( $\mathrm{ppm}$ vs time). Cylindrical pins of $10 \mathrm{~mm}$ in diameter and $30 \mathrm{~mm}$ long $(\approx 20 \mathrm{~g})$ were used to obtain the steel desorption curves.

A LECO DH603 hydrogen analyser, able to measure hydrogen concentrations between 0.1 and $2500 \mathrm{ppm}$, was used to measure the hydrogen content. The measuring principle of the equipment is based on the difference in thermal conductivity between a reference gas flow of pure nitrogen and a secondary flow composed of nitrogen and the hydrogen thermally extracted from the analysed specimen. This difference is measured by means of a Wheatstone bridge and converted into a signal that can be interpreted by the device's software, which finally gives the hydrogen concentration in ppm.

The procedure to obtain the desorption curves was the following. All the pins were removed from the liquid nitrogen at the same time and left in air at room temperature. Then, the hydrogen concentration of the different samples was measured at different time intervals. Before starting the measurement, each pin was cleaned in an ultrasonic bath with acetone for 5 minutes and carefully dried using cold air. The analysis to determine the hydrogen concentration consisted in keeping the sample at $1100^{\circ} \mathrm{C}$ for $400 \mathrm{~s}$.

\subsection{Tensile tests}

Tensile tests on both smooth and circumferentially-notched round-bar specimens, whose dimensions and geometries are shown in Fig. 1, were performed on an Instron 5582 tensile testing machine.
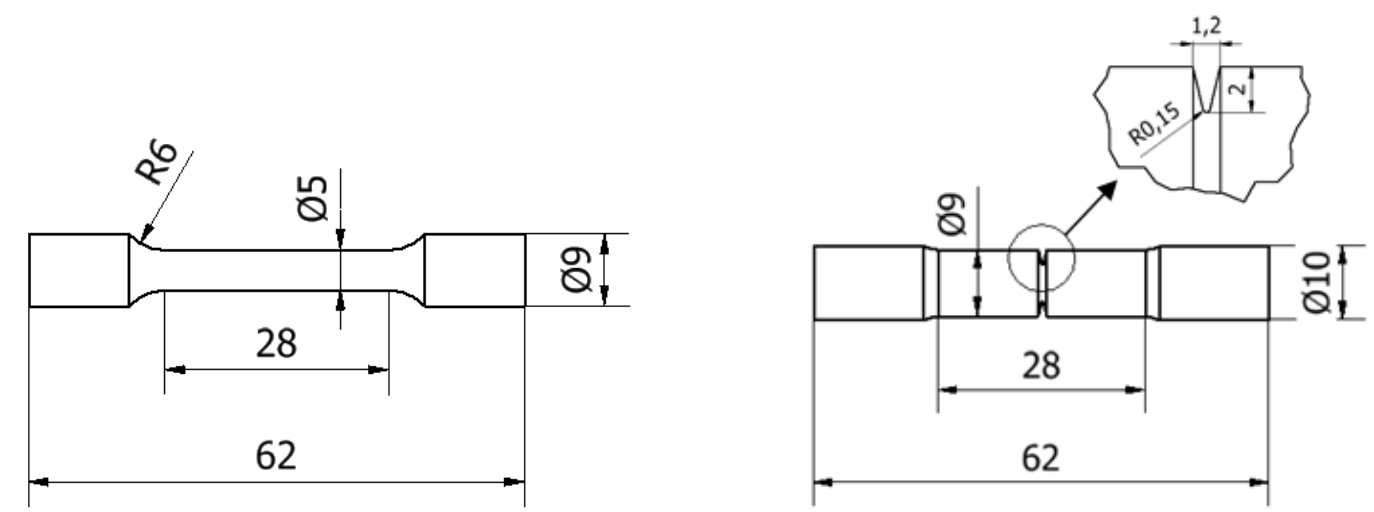

Fig. 1. Geometry and dimensions $(\mathrm{mm})$ of the smooth and notched specimens used in tensile tests.

The stress concentration factor of the notched tensile specimen was calculated according to Neuber [13]. Using a notch root radius of $0.15 \mathrm{~mm}$ and a radius of the notch cross-section of $5 \mathrm{~mm}$, a stress concentration factor $\mathrm{K}_{\mathrm{t}}=4.25$ was obtained. This value is in agreement with those calculated by other authors for similar specimen geometries [4, 14]. 
The behaviour of uncharged and hydrogen pre-charged specimens was compared in these tests. All uncharged tests were performed under a displacement rate of $0.4 \mathrm{~mm} / \mathrm{min}$, but different displacement rates, $0.4,0.04$ and $0.004 \mathrm{~mm} / \mathrm{min}$, were applied in the case of the pre-charged specimens to study the influence of this parameter on $\mathrm{HE}$.

In the case of the notched specimens, the engineering notched tensile strength $\left(\sigma_{\mathrm{N}}\right)$ was defined as the maximum tensile load divided by the initial cross-sectional area of the notch. The extent of hydrogen embrittlement was assessed by means of the embrittlement index (EI), defined in Equation 1 (EI varies from 0 , no embrittlement at all, to $100 \%$, maximum possible hydrogen embrittlement).

$$
E I[\%]=\frac{X-X_{H}}{X} \cdot 100
$$

where $\mathrm{X}$ and $\mathrm{X}_{\mathrm{H}}$ are respectively the measured steel property evaluated without and with hydrogen. The influence of the displacement rate on the reduction in area, RA, of the hydrogen pre-charged notched tensile specimens was also assessed.

\subsection{FEM simulation}

In order to fully characterize the geometry of the notched tensile specimens and discuss the obtained experimental results, the FEM software Abaqus CAE was used to obtain the stresses that developed around the notch region of the specimen at the moment of failure. A 2D elastic-plastic finite element model with a Hollomon hardening law obtained from the tensile tests was developed, since the specimens were axisymmetric (Fig.1). The geometry of the specimens was unevenly meshed, being this mesh finer in the vicinity of the notch root. 8-node biquadratic axisymmetric quadrilateral elements with reduced integration (CAX8R) were used with a minimum size around $40 \mu \mathrm{m}$.

\subsection{Observation of microstructures and fracture surfaces}

The microstructures obtained after the application of the different heat treatments were observed by means of a scanning electron microscope (SEM JEOL-JSM5600) under an acceleration voltage of $20 \mathrm{kV}$. The samples were previously ground, then polished with diamond paste, and finally etched with Nital-2\%. The fracture surfaces of the tested specimens were also analysed using the same scanning electron microscope under different magnifications.

\section{RESULTS}

\subsection{Steel microstructures}

The SEM microstructures of the 5 grades of 42CrMo steel are shown in Fig. 2 under 10000x magnification. The microstructure presented in Fig. 2 f corresponds to a quenched and non-tempered sample, in which a prior austenite grain size of $15-20 \mu \mathrm{m}$ was determined.

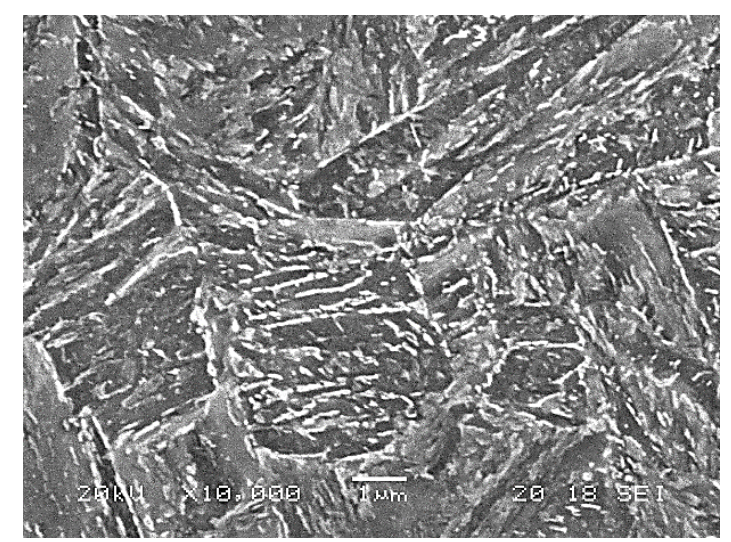

(a)

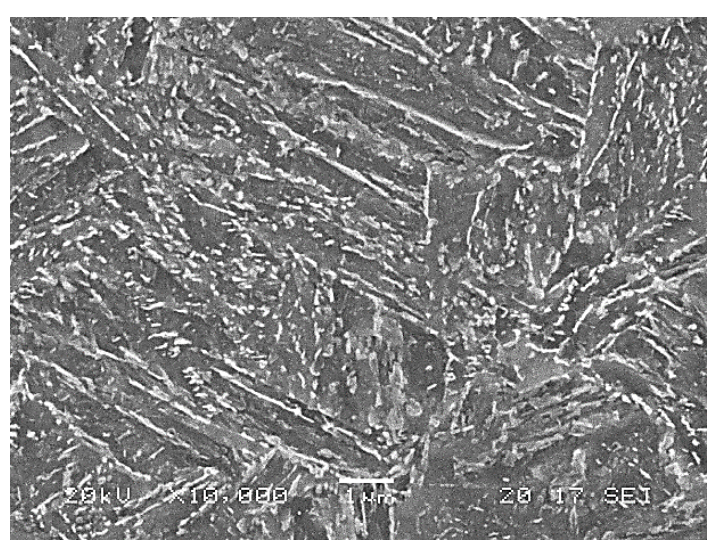

(b) 


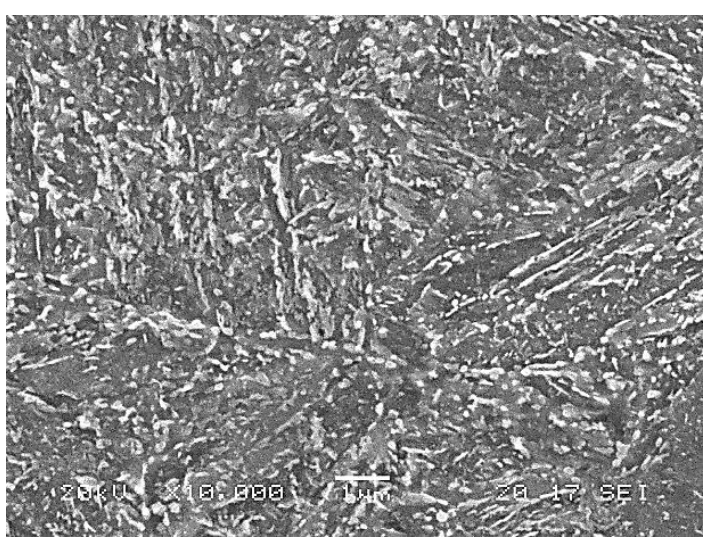

(c)

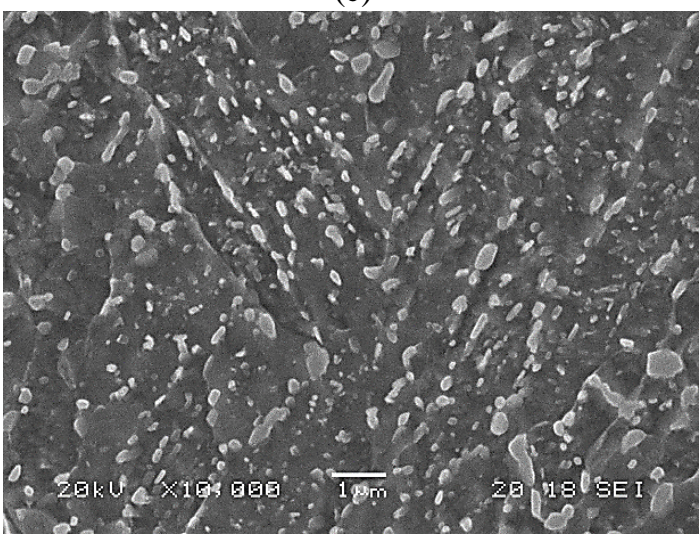

(e)

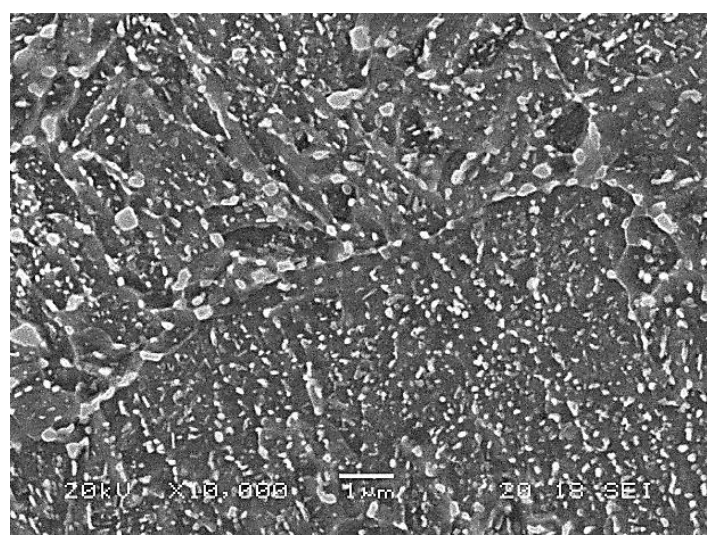

(d)

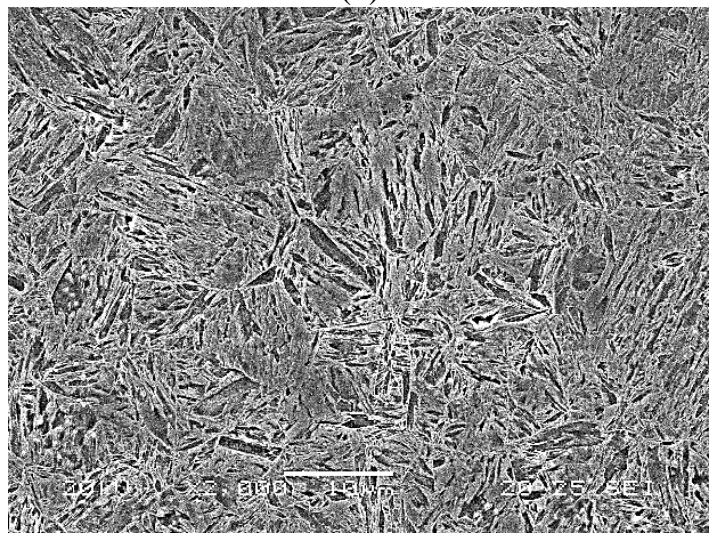

(f)

Fig. 2. SEM microstructures of $42 \mathrm{CrMo} 4$ steel, quenched and tempered at (a) $500^{\circ} \mathrm{C}$, (b) $550^{\circ} \mathrm{C}$, (c) $600^{\circ} \mathrm{C},(d) 650^{\circ} \mathrm{C}$, (e) $700^{\circ} \mathrm{C}$ and f) Quenched and non-tempered.

In all cases, the obtained microstructure was tempered martensite (the profuse carbide precipitation that takes place during the tempering stage can be seen). With increasing tempering temperature, microstructure acicularity decreases, internal residual stresses are released, dislocation rearrangement/annihilation and substructure recovery takes place, given rise to the decrease of martensite lath boundaries [15]. Furthermore, elongated carbides break up, globulize, and finally grow to yield a more uniform distribution.

The aforementioned microstructural differences give rise to the hardness and tensile properties listed in Table 3. As expected, Brinell hardness, HB, yield strength, $\sigma_{\mathrm{ys}}$, and ultimate tensile strength, $\sigma_{\mathrm{uts}}$, progressively decrease with increasing tempering temperature, while the opposite occurs with tensile elongation, $\varepsilon$, and reduction in area, RA.

\begin{tabular}{|c|c|c|c|c|c|c|}
\hline Steel Grades & Heat treatment & HB & $\begin{array}{c}\sigma_{y s} \\
{[\mathrm{MPa}]}\end{array}$ & $\begin{array}{c}\sigma_{\text {uts }} \\
{[\mathrm{MPa}]}\end{array}$ & $\begin{array}{c}\varepsilon \\
{[\%]}\end{array}$ & $\begin{array}{c}\text { RA } \\
{[\%]}\end{array}$ \\
\hline 42CrMo4_700 & $845^{\circ} \mathrm{C}+\mathrm{WQ}+\mathrm{T} 700^{\circ} \mathrm{C} / 2 \mathrm{~h}$ & 201 & 622 & 710 & 22.4 & 61.3 \\
\hline 42CrMo4_650 & $845^{\circ} \mathrm{C}+\mathrm{WQ}+\mathrm{T} 650^{\circ} \mathrm{C} / 2 \mathrm{~h}$ & 246 & 820 & 905 & 16.3 & 57.1 \\
\hline 42CrMo4_600 & $845^{\circ} \mathrm{C}+\mathrm{WQ}+\mathrm{T} 600^{\circ} \mathrm{C} / 2 \mathrm{~h}$ & 281 & 880 & 985 & 14.6 & 55.4 \\
\hline 42CrMo4_550 & $845^{\circ} \mathrm{C}+\mathrm{WQ}+\mathrm{T} 550^{\circ} \mathrm{C} / 2 \mathrm{~h}$ & 307 & 1023 & 1113 & 13.9 & 48.9 \\
\hline 42CrMo4_500 & $845^{\circ} \mathrm{C}+\mathrm{WQ}+\mathrm{T} 500^{\circ} \mathrm{C} / 2 \mathrm{~h}$ & 335 & 1086 & 1198 & 12.7 & 50.6 \\
\hline
\end{tabular}

Table 3. Hardness $(\mathrm{HB})$ and tensile properties of the different $42 \mathrm{CrMo} 4$ grades under study.

It is well known that hydrogen embrittlement increases with the strength level of the steel $[3,4]$, hence grades tempered at lower temperatures are expected to be more susceptible to HE.

\subsection{Hydrogen desorption curves}


The hydrogen desorption behaviour at room temperature of each $42 \mathrm{CrMo} 4$ steel grade is shown in Fig. 3. An initial hydrogen content, $\mathrm{C}_{\mathrm{H} 0}$, between 1.2 and $1.9 \mathrm{ppm}$ was introduced in the different steel grades. As the hydrogen content present in these grades before hydrogen charging was $0.2 \mathrm{ppm}$, a significant hydrogen uptake took always place. Hydrogen uptake increased with decreasing tempering temperature, as hydrogen microstructural trapping is greater in distorted high energy martensitic microstructures. The final hydrogen content, $\mathrm{C}_{\mathrm{Hf}}$, is the hydrogen strongly trapped and retained in the steel microstructure after a long time at room temperature. This value is even more dependent on the microstructure of the steel than the former value, decreasing as the tempering temperatures increases, mainly due to stress relaxation and the reduction in dislocation density and interphases (martensite laths, blocks and packets). Moreover, the diffusible hydrogen, i.e. the difference between these two values, $\mathrm{C}_{\mathrm{H} 0}-\mathrm{C}_{\mathrm{Hf}}$, is the hydrogen able to move freely within the steel at room temperature, overcome traps, and diffuse out of the steel. All the hydrogen values measured in the different $42 \mathrm{CrMo} 4$ steel grades are shown in Table 4. It is also worth noting that diffusible hydrogen progressively increases with increasing tempering temperature.

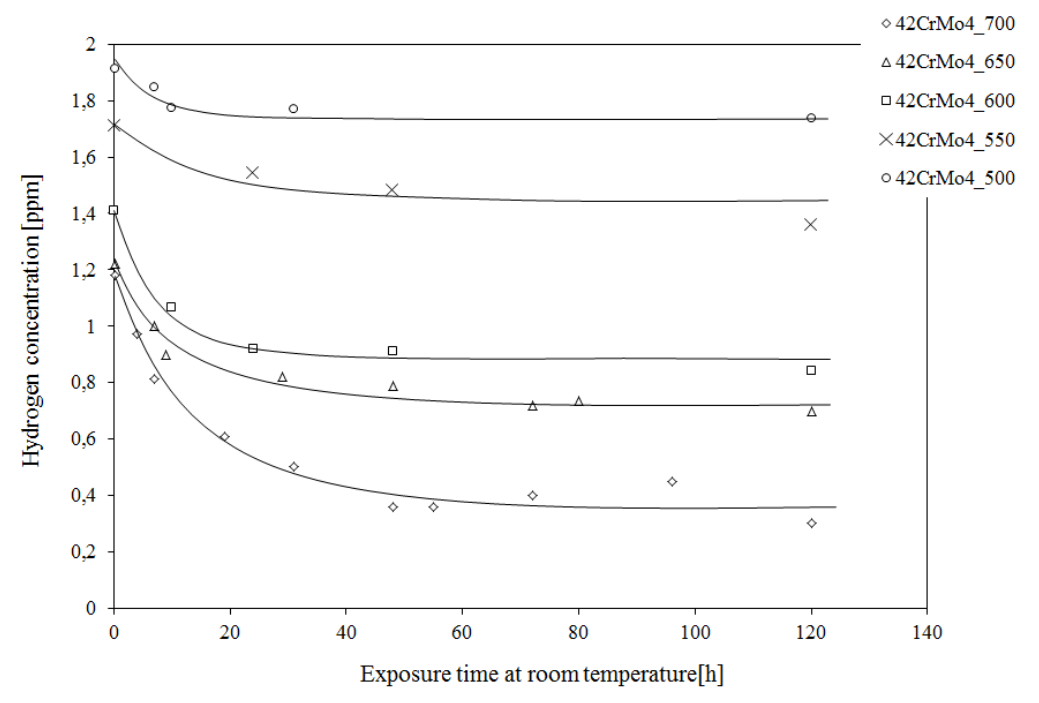

Fig. 3. Hydrogen desorption curves of 42CrMo4 steel grades at room temperature.

\begin{tabular}{|c|c|c|c|c|}
\hline Steel Grades & HB & $\mathrm{C}_{\mathrm{H} 0}[\mathrm{ppm}]$ & $\mathrm{C}_{\mathrm{Hf}}[\mathrm{ppm}]$ & $\mathrm{C}_{\mathrm{H} 0}-\mathrm{C}_{\mathrm{Hf}}[\mathrm{ppm}]$ \\
\hline 42CrMo4_700 & 201 & 1.2 & 0.4 & 0.8 \\
\hline 42CrMo4_650 & 246 & 1.2 & 0.7 & 0.5 \\
\hline 42CrMo4_600 & 281 & 1.4 & 0.9 & 0.5 \\
\hline 42CrMo4_550 & 307 & 1.7 & 1.4 & 0.3 \\
\hline 42CrMo4_500 & 335 & 1.9 & 1.7 & 0.2 \\
\hline
\end{tabular}

Table 4. Initial $\left(C_{H 0}\right)$, final $\left(C_{H f}\right.$, hydrogen retained after a month at room temperature) and diffusible hydrogen $\left(C_{H O}-C_{H f}\right)$ measured on the different $42 \mathrm{CrMo} 4$ steel grades.

\subsection{Tensile tests on smooth specimens}

Tensile tests were performed on smooth hydrogen pre-charged specimens of each grade of 42CrMo 4 steel. Fig. 4 shows the stress-strain curves obtained with grades tempered at the highest and lowest temperatures $\left(700\right.$ and $\left.500^{\circ} \mathrm{C}\right)$. No significant difference was observed between tests carried out at different displacement rates. Fig. 5 shows the embrittlement index (EI) corresponding to fracture strength and reduction in area versus steel hardness for the lowest displacement rate used in these tests $(0.04 \mathrm{~mm} / \mathrm{min})$. A small reduction of the yield strength was also observed in the presence of internal hydrogen. 


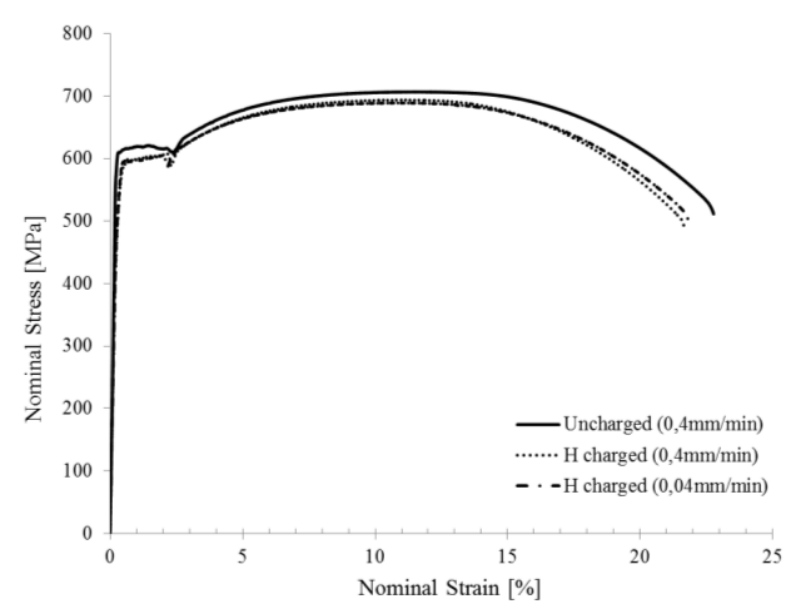

(a)

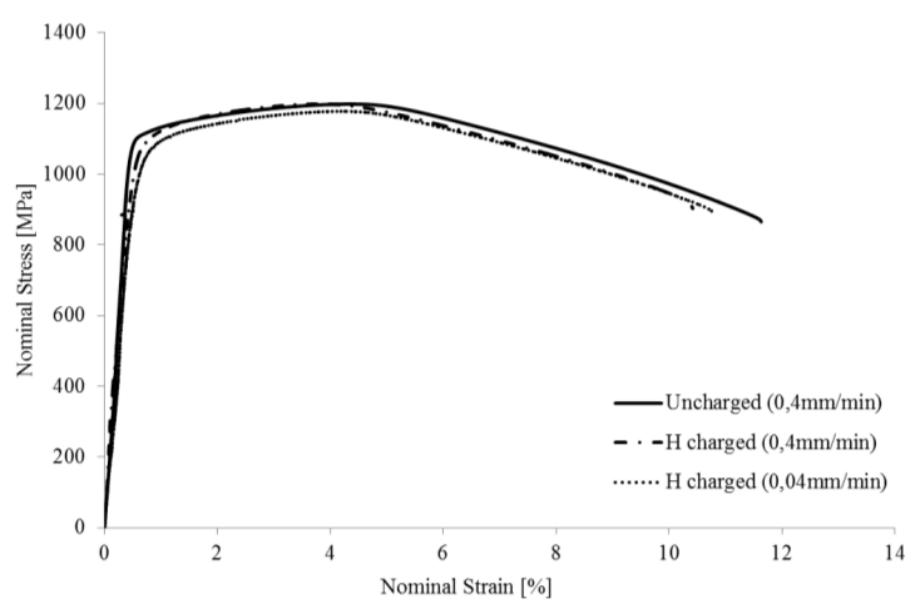

(b)

Fig. 4. Stress-strain curves of tensile tests, a) 42CrMo4_700 and b) 42CrMo4_500.

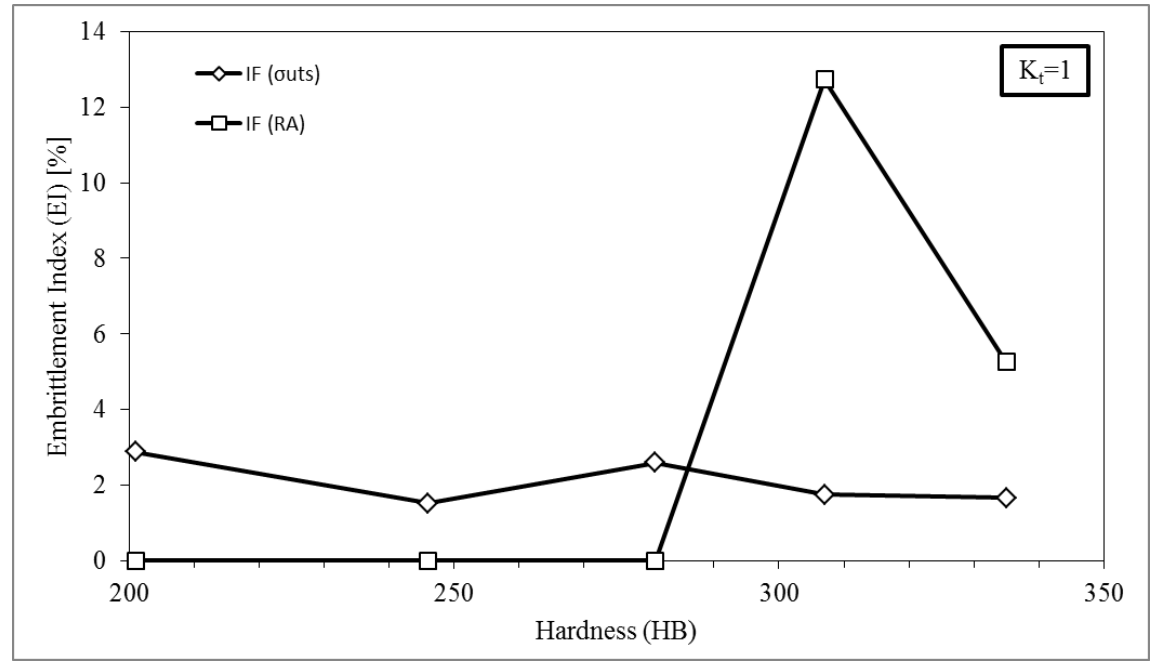

Fig. 5. Influence of tempering temperature on the embrittlement index (EI) calculated for fracture strength and reduction in area of hydrogen pre-charged tensile smooth specimens. Displacement rate: $0.04 \mathrm{~mm} / \mathrm{min}$.

The embrittlement index for strength remains almost constant at approximately $2 \%$, which means that hydrogen has little influence on the mechanical strength for this geometry, irrespective of the hardness of the steel. Regarding the reduction in area, the EI is $0 \%$ for tempering temperatures greater than $600^{\circ} \mathrm{C}$, increasing slightly to 6-12\% for the two lowest temperatures (i.e. the hardest grades).

In conclusion, it may be stated that tensile properties measured on smooth specimens pre-charged with gaseous hydrogen remained practically unaffected, irrespective of the applied displacement rate. Moreover, the fracture micromechanism observed on the failure surfaces of all these specimens under the scanning electron microscope was always fully ductile (microvoid coalescence).

\subsection{Tensile tests on notched specimens}

Fig. 6 shows the results obtained for $42 \mathrm{CrMo} 4$ quenched and tempered at 500 and $700^{\circ} \mathrm{C}$, which were respectively the grades less and most affected by the deleterious effects of hydrogen. The stress-strain curves of the uncharged (displacement rate of $0.4 \mathrm{~mm} / \mathrm{min}$ ) and hydrogen pre-charged notched specimens, tested at different displacement rates $(0.4,0.04$ and $0.004 \mathrm{~mm} / \mathrm{min})$, are shown in Fig. 6 . All these graphs show a reduced plastic region, as most of the specimen is only elastically strained, except for a very small region close to the stress concentrator, as it will be demonstrated in the next section. 
As the test displacement rate decreases, it can be seen that the damage caused by hydrogen increases considerably in the case of the steel grade tempered at $500^{\circ} \mathrm{C}\left(\sigma_{\mathrm{ys}}=1086 \mathrm{MPa}\right)$. In fact, the fracture strength of this steel grade decreased almost $60 \%$, and the reduction in area more than $90 \%$, when specimen was pre-charged and tested at the lowest strain rate.

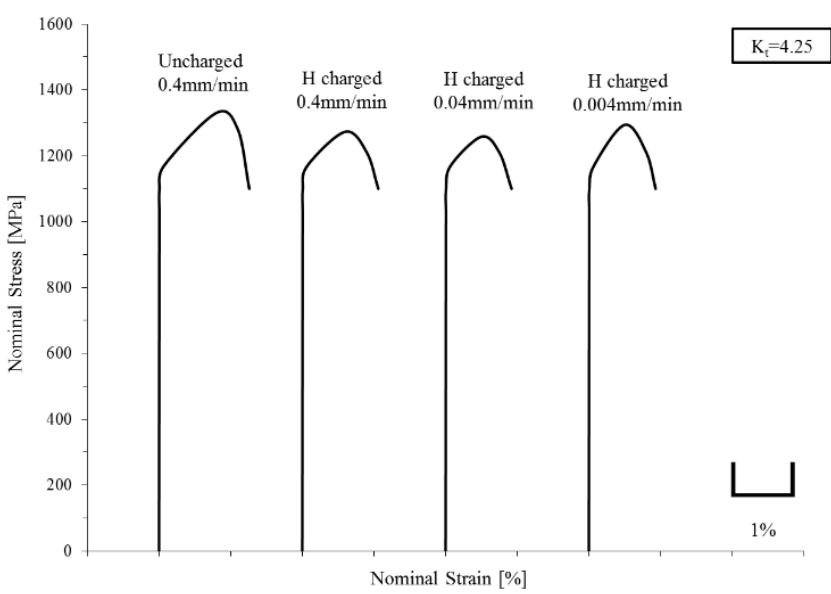

(a)

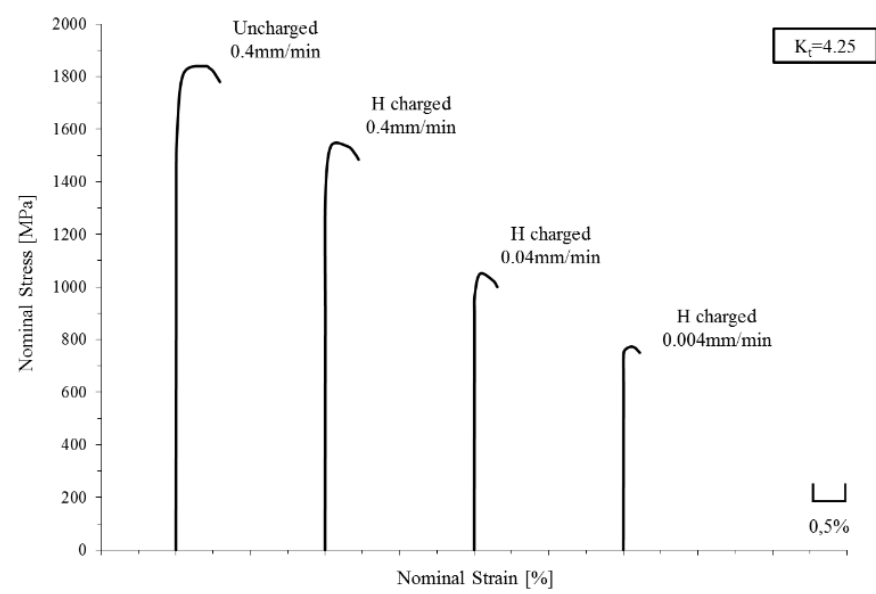

(b)

Fig. 6. Stress-strain curves of the uncharged and hydrogen pre-charged notched tensile specimens $\left(K_{t}=4.25\right)$ of a) 42CrMo4_700 and b) 42CrMo4_500 steel. Influence of the applied displacement rate.

Quite different behaviour was observed on the 42CrMo4_700 grade. In this case, due to the much lower yield strength of the steel $\left(\sigma_{y s}=622 \mathrm{MPa}\right)$, the effects of hydrogen were rather slight. Furthermore, the displacement rate does not seem to play an important role in this case. According to Fig. 3, the amount of pre-charged hydrogen in this steel grade was also the lowest (1.2 ppm). Due to this fact and its low yield strength, hydrogen accumulation in the notched region of this steel grade was limited and the embrittlement reaction was minimised. The steel submitted to the other tempering temperatures gave rise to intermediate behaviours.

Table 5 summarizes the results obtained in the tensile tests performed on the notched specimens for the different $42 \mathrm{CrMo} 4$ steel grades. The values of the notch tensile strength, $\sigma_{\mathrm{N}}$, and reduction in area, RA, are shown for each grade, uncharged and hydrogen pre-charged under different displacement rates. Embrittlement indexes calculated for the aforementioned mechanical properties and prevailing fracture micromechanisms are also presented in Table 5. Moreover, the test duration and approximate average hydrogen content, $\mathrm{C}_{\mathrm{H}}$, of the specimens in the course of the tests can also be seen in the same table. It can be seen that test duration barely affected the hydrogen content in these specimens, except for the lowest displacement rate. Anyway, the values of $\mathrm{C}_{\mathrm{H}}$ in Table 5 may be taken with caution as they were derived from the desorption curves (Fig. 3) obtained with samples with slightly different dimensions.

Fig. 7 shows the notch tensile strength and reduction in area obtained with all these grades using uncharged and hydrogen pre-charged notched tensile specimens (under a displacement rate of $0.004 \mathrm{~mm} / \mathrm{min}$ ). The presence of hydrogen barely modifies the notched tensile strength of the grades with the lowest hardness, 201 and $246 \mathrm{HB}$ (respectively tempered at 700 and $650^{\circ} \mathrm{C}$ ), but causes a significant decrease in the case of the grades with the highest hardness (tempered at the lowest temperatures). This effect greatly increases with increasing hardness (i.e. decreasing tempering temperature). In the case of the reduction in area, the behaviour is different: the embrittlement effect of hydrogen is evident and practically constant for all the tested grades. 


\begin{tabular}{|c|c|c|c|c|c|c|c|c|}
\hline Steel Grade & $\begin{array}{c}\text { Test } \\
\text { speed } \\
{[\mathrm{mm} / \mathrm{min}]}\end{array}$ & $\begin{array}{c}\text { Test } \\
\text { duration }\end{array}$ & $\begin{array}{c}\mathrm{C}_{\mathrm{H}} \\
{[\mathrm{ppm}]}\end{array}$ & $\begin{array}{c}\sigma_{\mathrm{N}} \\
{[\mathrm{MPa}]}\end{array}$ & $\begin{array}{l}\text { RA } \\
{[\%]}\end{array}$ & $\begin{array}{c}\mathrm{EI}\left(\sigma_{\mathrm{uts}}\right) \\
{[\%]}\end{array}$ & $\begin{array}{c}\mathrm{EI}(\mathrm{RA}) \\
{[\%]}\end{array}$ & $\begin{array}{c}\text { Fracture } \\
\text { Micromechanisms }\end{array}$ \\
\hline \multirow{4}{*}{$\begin{array}{l}\text { 42CrMo4_700 } \\
(201 \mathrm{HB})\end{array}$} & 0.4 & $12 \mathrm{~min}$ & Uncharged & 1330 & 12.5 & - & - & MVC \\
\hline & 0.4 & $9 \min$ & 1.2 & 1273 & 11.8 & 4 & 6 & MVC \\
\hline & 0.04 & $80 \mathrm{~min}$ & 1.2 & 1258 & 10.3 & 5 & 18 & MVC \\
\hline & 0.004 & $14 \mathrm{~h}$ & $1.2 \rightarrow 0.7$ & 1294 & 9.6 & 3 & 23 & MVC \\
\hline \multirow{4}{*}{$\begin{array}{l}\text { 42CrMo4_650 } \\
(246 \mathrm{HB})\end{array}$} & 0.4 & $17 \mathrm{~min}$ & Uncharged & 1582 & 11.9 & - & - & MVC \\
\hline & 0.4 & $15 \mathrm{~min}$ & 1.2 & 1527 & 11.8 & 3 & 1 & MVC \\
\hline & 0.04 & $105 \mathrm{~min}$ & 1.2 & 1508 & 8.9 & 5 & 25 & MVC+PRHIC \\
\hline & 0.004 & $16 \mathrm{~h}$ & $1.2 \rightarrow 0.85$ & 1487 & 7.9 & 6 & 34 & MVC+PRHIC \\
\hline \multirow{4}{*}{$\begin{array}{l}\text { 42CrMo4_600 } \\
\text { (281HB) }\end{array}$} & 0.4 & $12 \mathrm{~min}$ & Uncharged & 1724 & 8.5 & - & - & MVC \\
\hline & 0.4 & $12 \mathrm{~min}$ & 1.4 & 1604 & 7.6 & 7 & 11 & MVC \\
\hline & 0.04 & $85 \mathrm{~min}$ & 1.4 & 1384 & 6.0 & 20 & 29 & MVC+PRHIC \\
\hline & 0.004 & $14 \mathrm{~h}$ & $1.4 \rightarrow 1.0$ & 1336 & 3.4 & 23 & 60 & $\mathrm{MVC}+\mathrm{PRHIC}+\mathrm{IG}$ \\
\hline \multirow{4}{*}{$\begin{array}{c}\text { 42CrMo4_550 } \\
(307 \mathrm{HB})\end{array}$} & 0.4 & $8 \mathrm{~min}$ & Uncharged & 1800 & 7.5 & - & - & MVC \\
\hline & 0.4 & $9 \min$ & 1.7 & 1602 & 6.3 & 11 & 16 & $\mathrm{MVC}+\mathrm{PRHIC}+\mathrm{IG}$ \\
\hline & 0.04 & $65 \mathrm{~min}$ & 1.7 & 1303 & 4.9 & 28 & 35 & $\mathrm{MVC}+\mathrm{PRHIC}+\mathrm{IG}$ \\
\hline & 0.004 & $11 \mathrm{~h}$ & $1.7 \rightarrow 1.6$ & 1248 & 2.7 & 31 & 64 & $\mathrm{MVC}+\mathrm{PRHIC}+\mathrm{IG}$ \\
\hline \multirow{4}{*}{$\begin{array}{l}\text { 42CrMo4_500 } \\
(335 \mathrm{HB})\end{array}$} & 0.4 & $15 \mathrm{~min}$ & Uncharged & 1833 & 6.4 & - & - & MVC \\
\hline & 0.4 & $13 \mathrm{~min}$ & 1.9 & 1534 & 4.7 & 16 & 27 & $\mathrm{MVC}+\mathrm{PRHIC}+\mathrm{IG}$ \\
\hline & 0.04 & $80 \mathrm{~min}$ & 1.9 & 1050 & 3.1 & 43 & 51 & $\mathrm{MVC}+\mathrm{PRHIC}+\mathrm{IG}$ \\
\hline & 0.004 & $6 \mathrm{~h}$ & $1.9 \rightarrow 1.8$ & 771 & 0.5 & 58 & 92 & $\mathrm{MVC}+\mathrm{PRHIC}+\mathrm{IG}$ \\
\hline
\end{tabular}

Table 5. Results of tensile tests performed on notched specimens $\left(K_{t}=4.25\right)$ of $42 \mathrm{CrMo} 4$ steel uncharged and hydrogen pre-charged and loaded at different displacement rates.

MVC: microvoid coalescence; PRHIC: plasticity-related hydrogen induced cracking; IG: intergranular.

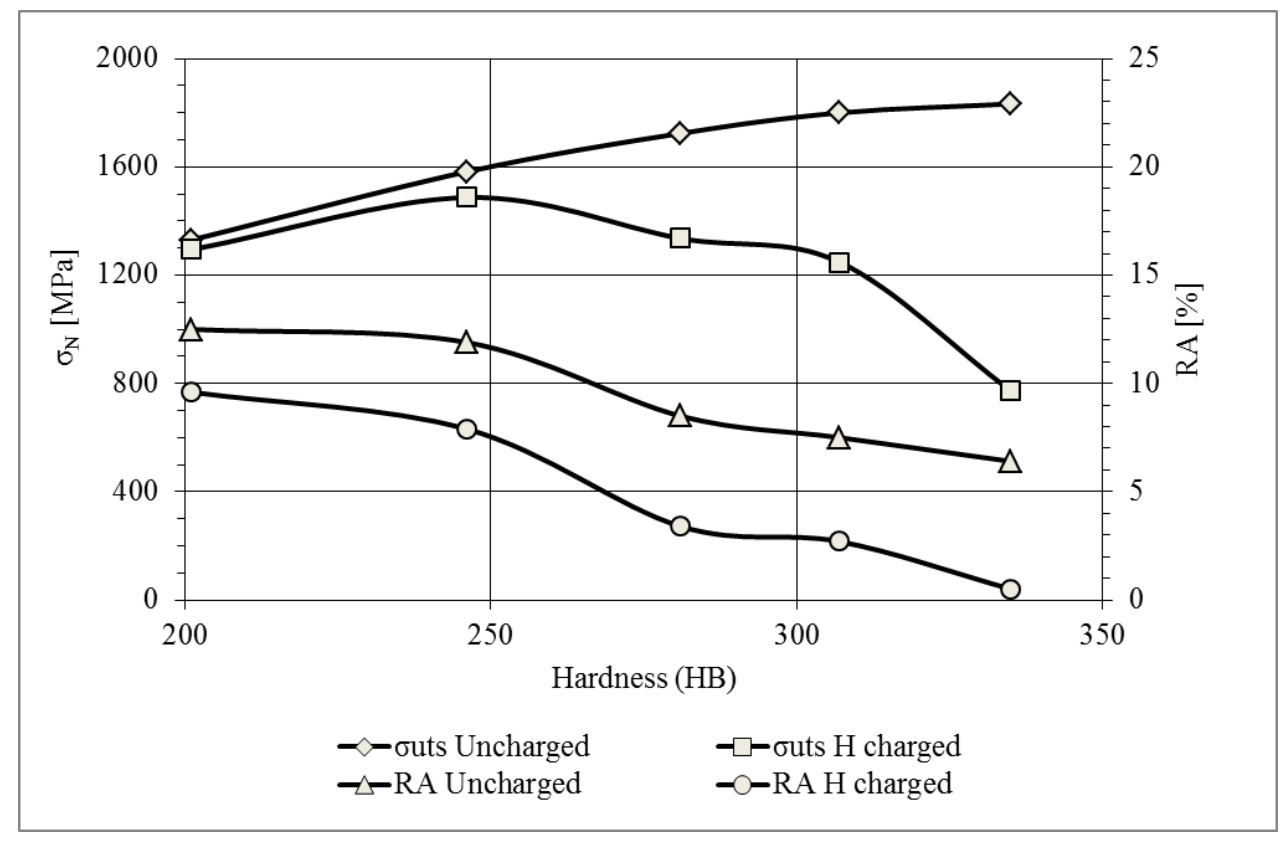

Fig. 7. Tensile strength and reduction in area versus hardness $(H B)$ of notched specimens $\left(K_{t}=4.25\right)$ without hydrogen and hydrogen pre-charged. Displacement rate: $0.004 \mathrm{~mm} / \mathrm{min}$.

\subsection{Fracture surfaces analysis of notched specimens}

The fracture surfaces corresponding to the notched specimens of the different steel grades under study were analysed using SEM and the main operative fracture micromechanisms were identified. 
The fracture micromechanism of all the tested grades without hydrogen was fully ductile, microvoid coalescence (MVC), as already shown in Table 5 and as can also be seen in Fig. 8 (a) and (b).

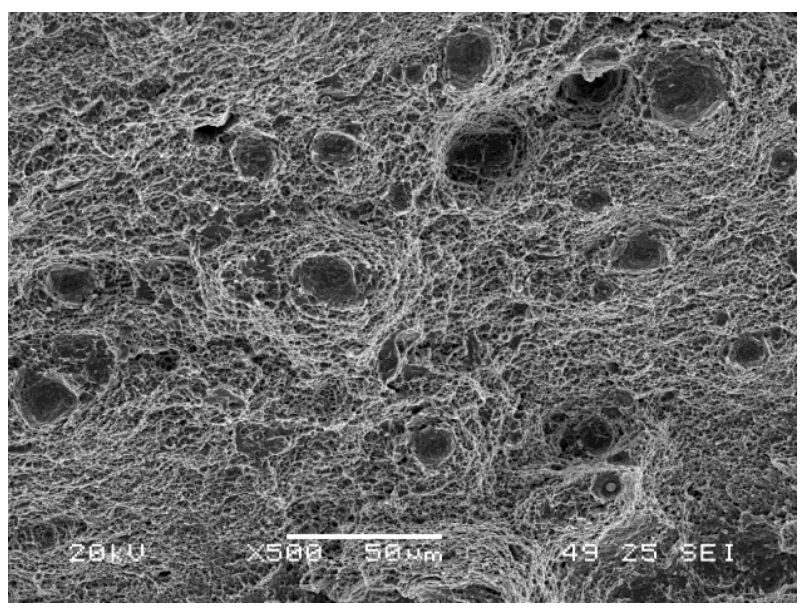

(a) 42CrMo4_700 (uncharged)

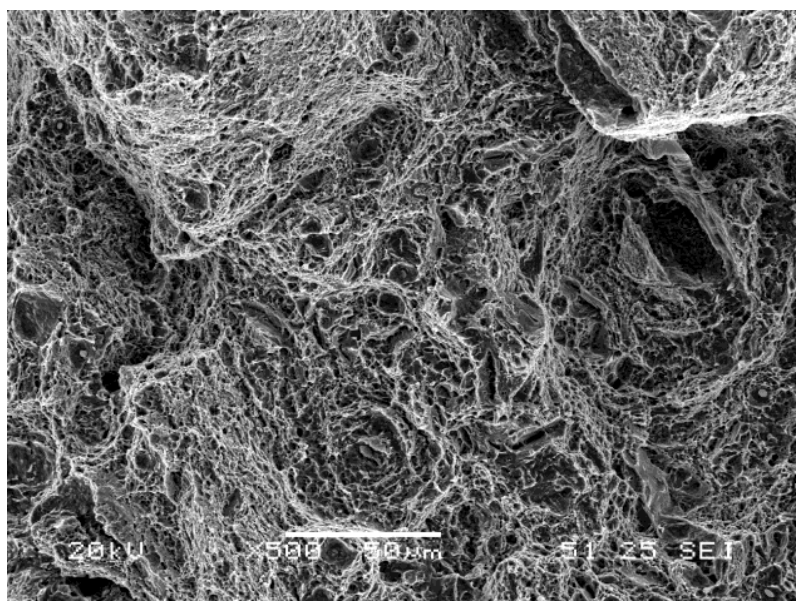

(b) 42CrMo4_500 (uncharged)

Fig. 8. Fracture micromechanism of (a) 42CrMo4_700 (uncharged) and (b)42CrMo4_500(uncharged). 500x.

The remaining fractographs, shown in Fig. 9, correspond to hydrogen pre-charged notched tensile specimens tested under displacement rates of $0.004 \mathrm{~mm} / \mathrm{min}$. Fig. 9 (a) corresponds to the grade tempered at $700^{\circ} \mathrm{C}$. The fracture micromechanism did not change in this grade, but elongated, flat areas such as those shown in Fig. 9 (a), were noted as a characteristic feature.

Furthermore, in the case of 42CrMo4_650, as can be seen in Fig. 9 (b) and (c), two different regions were observed in the specimen: a central region with microvoids, some of which were also enlarged, and a narrow peripheral region of around $100 \mu \mathrm{m}$ that exhibited the appearance of a transgranular fracture surface or quasi-cleavage, usually referred to as plasticity-related hydrogen induced cracking (PRHIC) in martensitic steels [16]. The PRHIC mechanism was first described by Takeda and McMahon [17] in reference to the fracture mechanism observed in a low alloy quenched and tempered steel in hydrogen gas. It is sometimes called tearing topography surface or TTS, which is described as a fracture surface characterised by ductile micro-plastic tearing on a very fine scale, along martensite lath interphases $[16,18]$. The appearance of this region can be better seen in Figs. 9 (d). The size of the characteristic features observed in Fig. 9 (d) are comparable to the microstructure units (martensite blocks) shown in Fig. 2 (d); hence, hydrogen accumulation promotes plastic deformation and final failure of these interphases.

As to the grades tempered at lower temperatures (600,550 and 500), the observed fracture micromechanism was a mixture of MVC, PRHIC and intergranular fracture (IG). In the bulk of the specimen, MVC was the predominant micromechanism, although PRHIC and IG were the characteristic micromechanisms in the periphery of these specimens and also, in some cases, along some radial "paths" leading from the periphery to the centre of the specimen. Examples of these characteristic surfaces are shown in Figs. 9 (e, f, g, h, i and j) at 500, 1000 and 2000x magnification.

It is worth noting that the width of the belt characterized by PRHIC and IG fracture increases with increasing hardness of the steel (i.e. decreasing tempering temperature), being greater than $250 \mu \mathrm{m}$ in all cases. Furthermore, the extent of intergranular fracture is larger in the grade with the highest strength, 42CrMo4_500, which also gave rise to the highest embrittlement indexes, as was seen in Table 5. The estimated size of the grains directly measured on these fractographs was around 15-20 $\mu \mathrm{m}$, which corresponds to the prior austenite grain size measured on the polished and etched sections of these steels (see Fig. 2 f). 


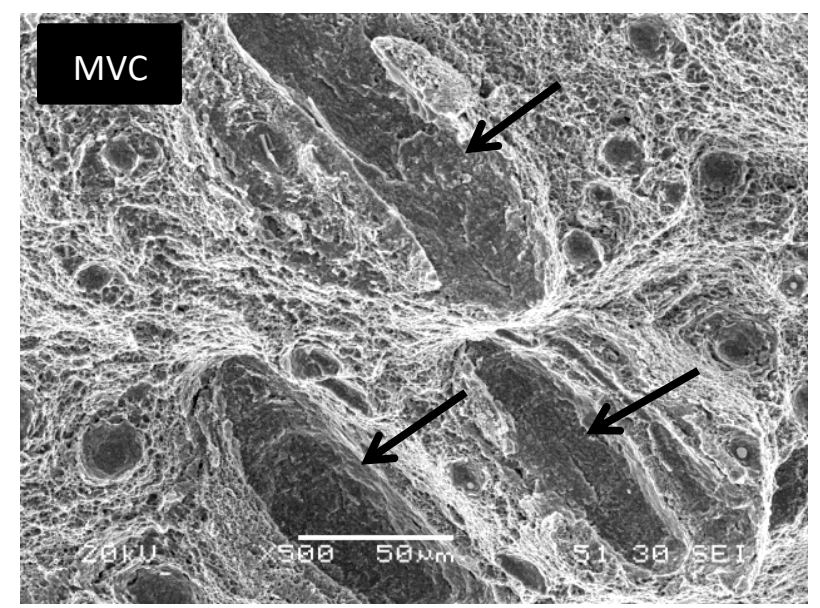

(a) 42CrMo4_700 (H pre-charged, 500x)
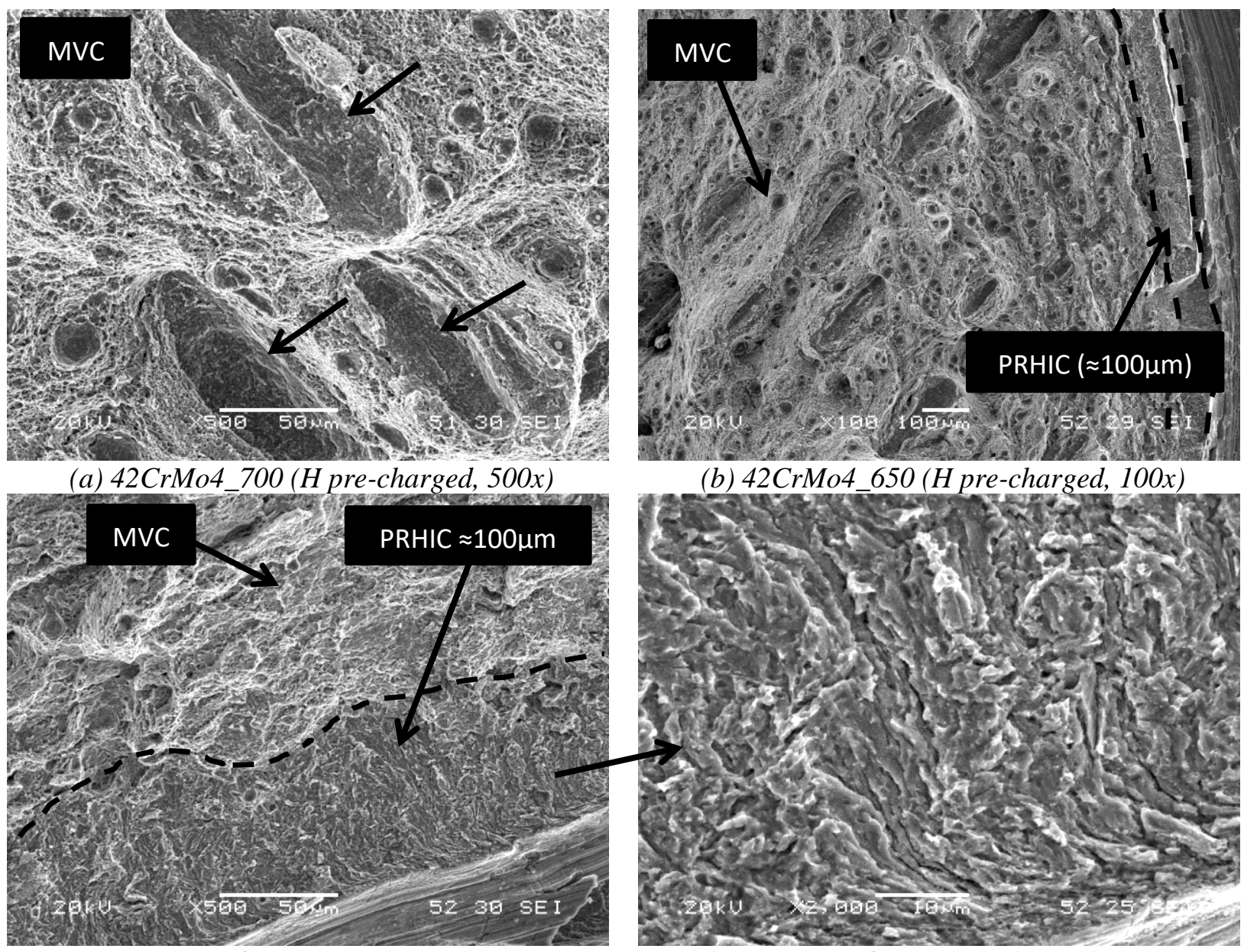

(b) 42CrMo4_650 (H pre-charged, 100x

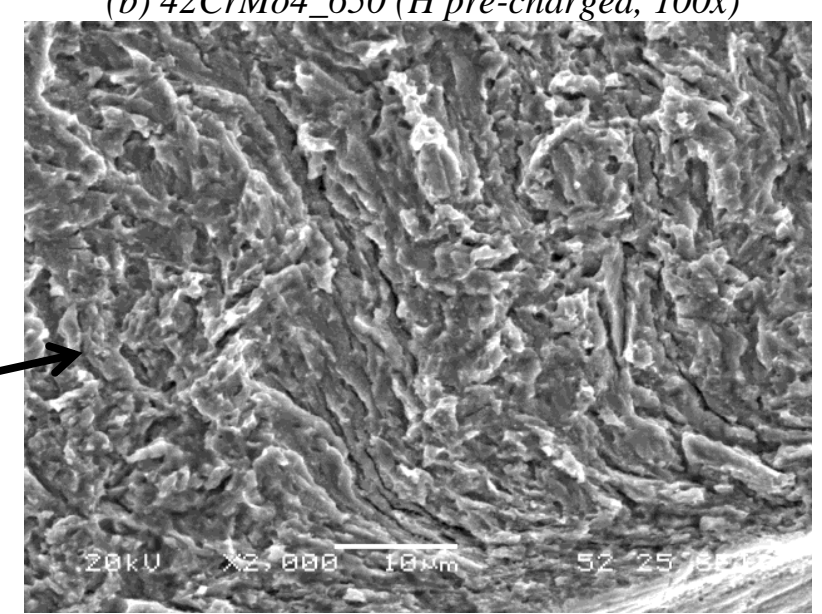

(c) 42CrMo4_650(H pre-charged, 500x)

(d) 42CrMo4_650 (H pre-charged, 2000x)

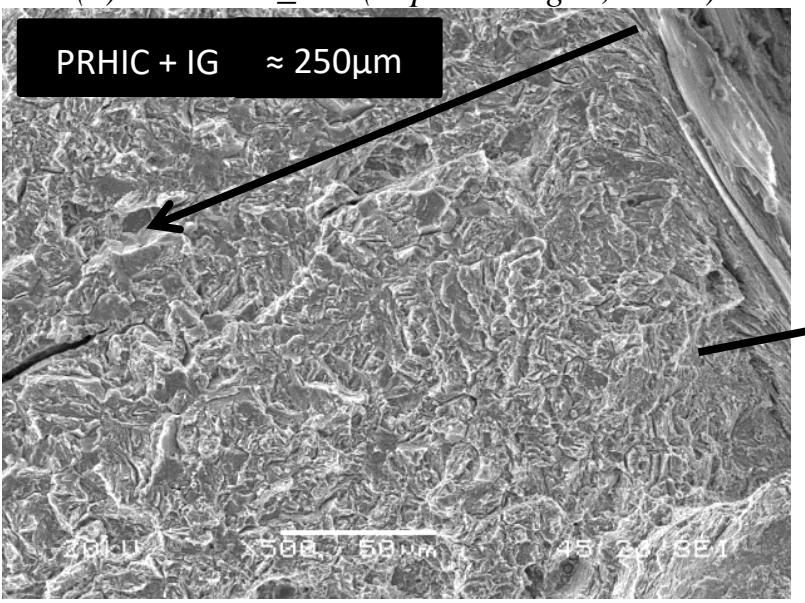

(e) 42CrMo4_600 (H pre-charged, 500x)

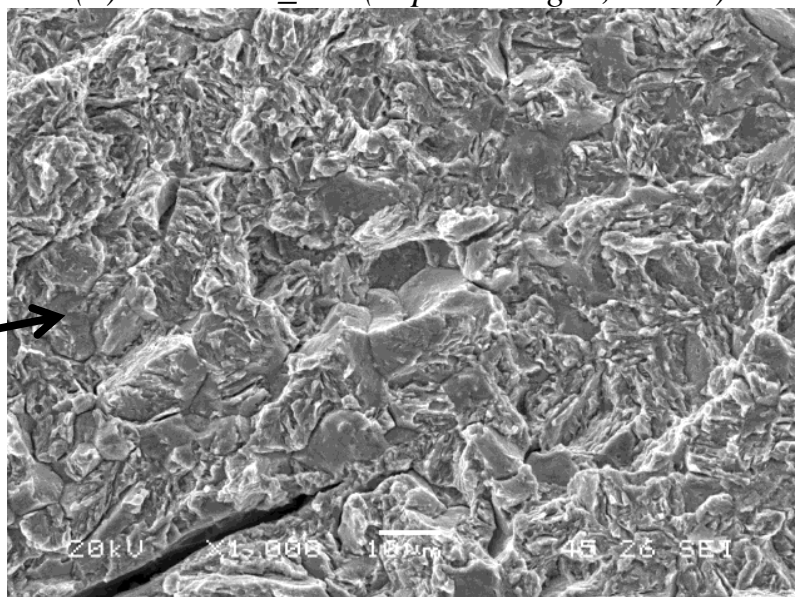

(f) 42CrMo4_600 (H pre-charged, 1000x) 


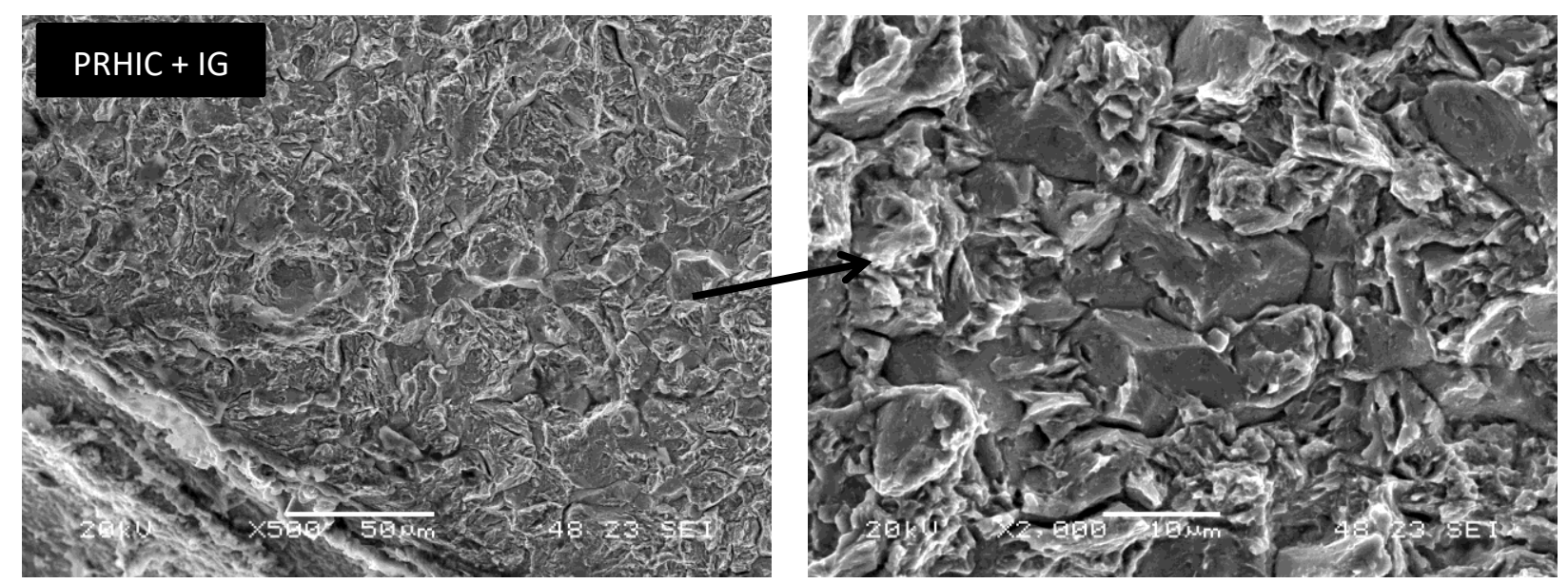

(g) 42CrMo4_550 (H pre-charged, 500x)

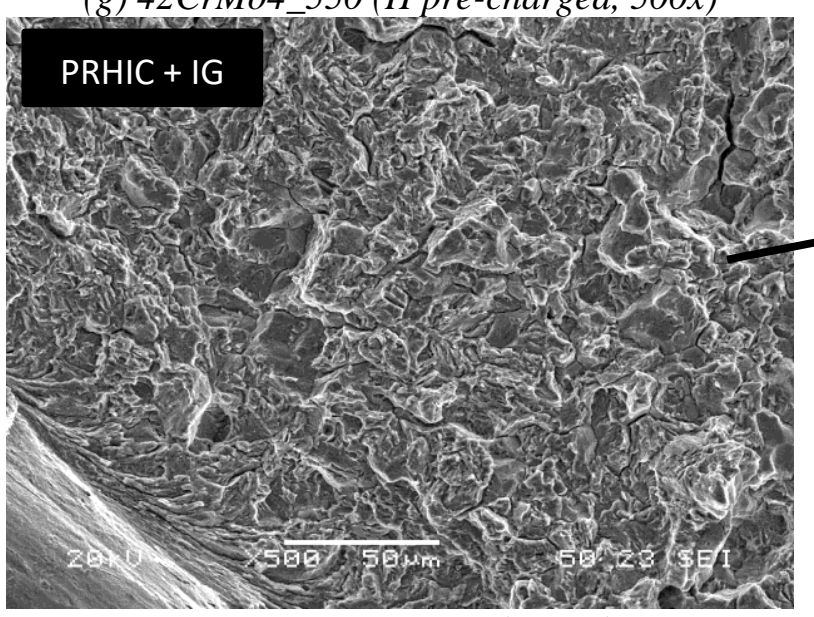

(i) 42CrMo4_500 (H pre-charged, 500x)

(h) 42CrMo4_550 (H pre-charged, 2000x)

Fig 9. Fracture micromechanisms of $42 \mathrm{CrMo} 4$ grades tested with hydrogen at a displacement rate of $0.004 \mathrm{~mm} / \mathrm{min}$.

Now, with the aim of explaining the aforementioned experimental results, the distribution of the local normal stress, perpendicular to the notch plane, $\sigma_{22}$, the Von Mises stress, $\sigma_{\mathrm{VM}}$ (Eq. 2), and the hydrostatic stress, $\sigma_{\mathrm{h}}$ (Eq. 3) was simulated along a radial path, defined from the free surface at the tip of the notch $(x=0)$ to the centre of the specimen in the notched section $(x=2.5 \mathrm{~mm})$.

$$
\begin{gathered}
\sigma_{V M}=\sqrt{\frac{\left(\sigma_{11}-\sigma_{22}\right)^{2}+\left(\sigma_{22}-\sigma_{33}\right)^{2}+\left(\sigma_{33}-\sigma_{11}\right)^{2}}{2}} \\
\sigma_{h}=\frac{\sigma_{11}+\sigma_{22}+\sigma_{33}}{3}
\end{gathered}
$$

being $\sigma_{11}, \sigma_{22}$ and $\sigma_{33}$ the principal stresses.

The $\sigma_{\mathrm{VM}} / \sigma_{\mathrm{ys}}$ ratio versus distance from notch tip at an applied stress equal to the notch tensile strength corresponding to the five analysed steel grades is shown in Fig. 10. The extension of the plastic region varies between 100 and $180 \mu \mathrm{m}$, increasing when decreasing the yield strength of the steel (higher tempering temperature). It is then worth noting that in all cases most of the specimen is only elastically strained, except for a very small region close to the stress concentrator. 


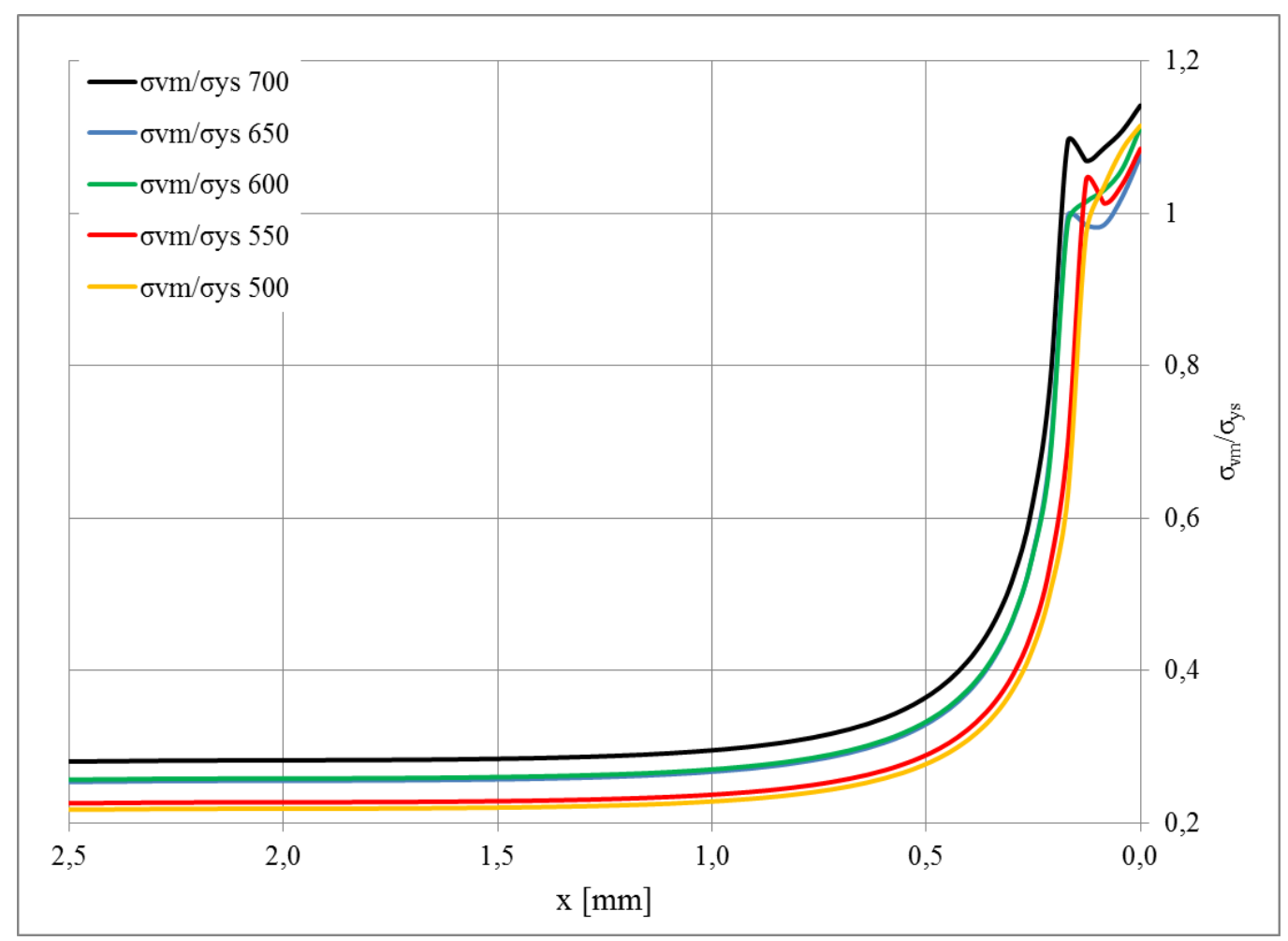

Fig. 10. Distribution of $\sigma_{V M} / \sigma_{y s}$ along the radial direction when the applied net stress is equal to the notch tensile strength. $42 \mathrm{CrMo} 4$ steel quenched and tempered at different temperatures.

Fig. 11 shows the evolution of $\sigma_{\mathrm{VM}}, \sigma_{\mathrm{h}}$, and $\sigma_{22}$ in the course of loading for one of the steel grades (42CrMo4_650). The two lowest stress profiles correspond to essentially full elastic loadings $\left(\sigma_{\mathrm{VM}} \leq \sigma_{\mathrm{ys}}=\right.$ $880 \mathrm{MPa}$ ). For higher applied loads, a plastic zone develops in the front of the notch and the last represented stress profile corresponds to the instant of failure of the specimen. It is also noted that once a plastic zone is developed $\left(\sigma_{\mathrm{VM}}>\sigma_{\mathrm{ys}}\right.$ ), a maximum in the Von Mises and in the hydrostatic stress profiles is produced at a certain depth of the notch tip and the location of this maximum displaces to larger depths when loading is increased. 

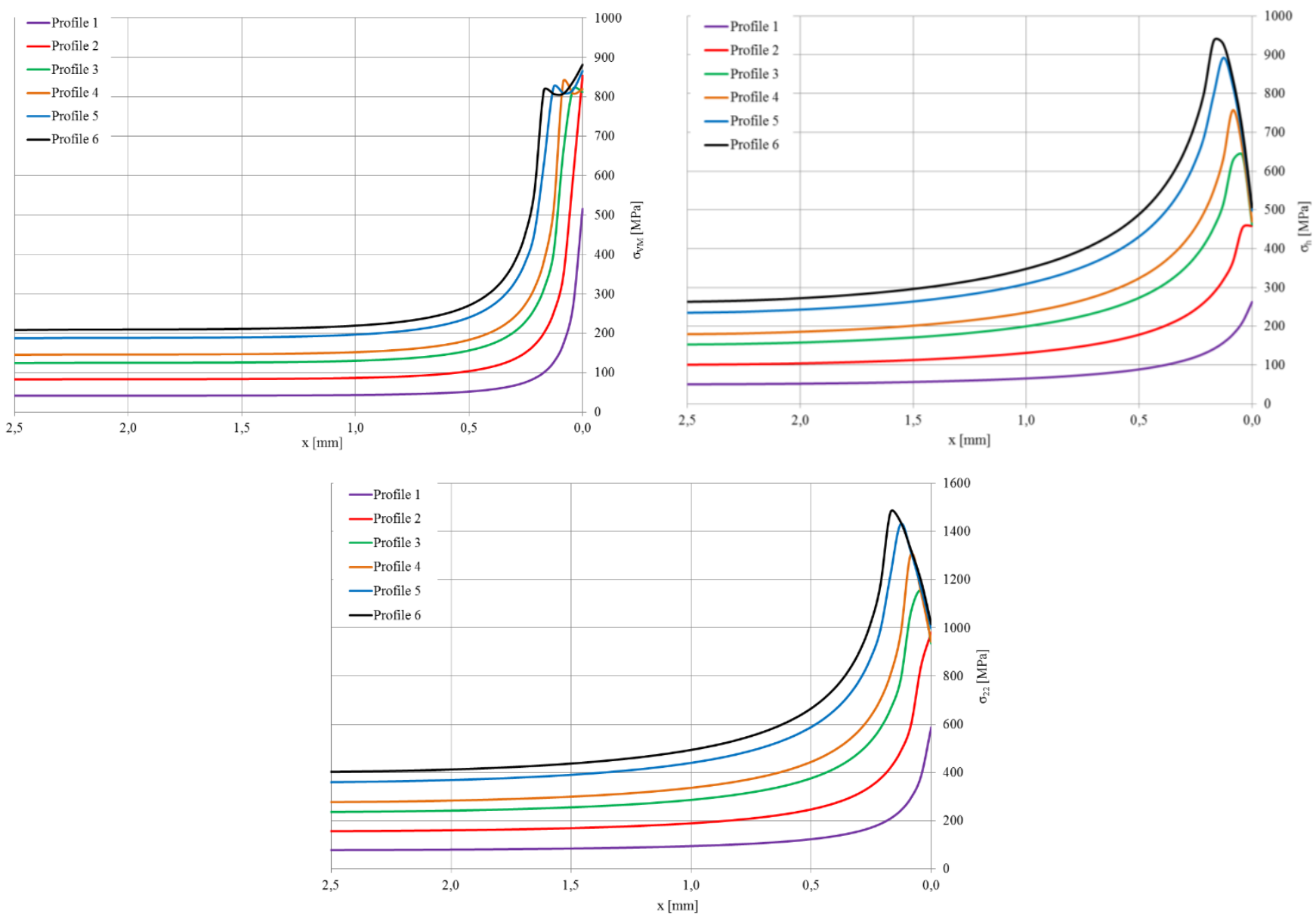

Fig. 11. Distribution of $\sigma_{V M}, \sigma_{h}$ and $\sigma_{22}$ along the radial direction for six different loading steps. 42CrMo4_650. Applied load increases from 1 to 6.

Fig. 12 shows now the evolution of $\sigma_{\mathrm{h}}$, and $\sigma_{22}$ versus distance from the notch tip at an applied stress equal to the notch tensile strength corresponding to the five analysed steel grades. The level of the normal stress and hydrostatic stress increases, especially in the region closer to the notch tip, as the yield strength of the steel grade is increased and at the same time it also worth noting the displacement of the maximum of both aforementioned stresses to a lower depth.
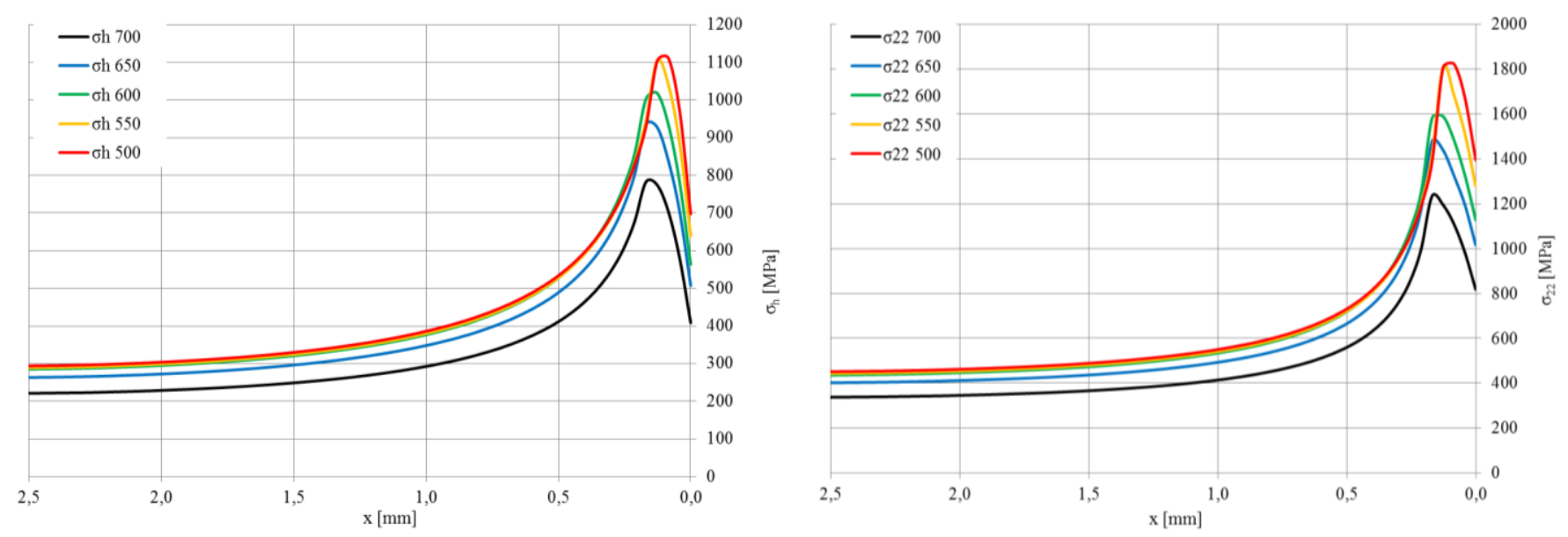

Fig. 12. Distribution of $\sigma_{h}$, and $\sigma_{22}$ along the radial direction when the applied net stress is equal to the notch tensile strength. 42CrMo4 steel quenched and tempered at different temperatures. 


\section{Discussion}

It is well known that the internal hydrogen distribution in high strength steels submitted to external loads is governed by hydrostatic stress [17]. Due to this effect, hydrogen atoms diffuse along the steel microstructure and move to regions submitted to the highest hydrostatic stress, located near the notch tip (see Figs. 11 and 12). As a result of the accumulation of hydrogen in this region, a critical hydrogen concentration may be reached, leading to premature failure of the specimen [19].

As a summary of the study, Fig. 13 shows the embrittlement indexes under a displacement rate of 0.004 $\mathrm{mm} / \mathrm{min}$ calculated for the notch tensile strength and the reduction in area versus the Brinell hardness of each grade (tempering temperature). Additionally, the fracture micromechanisms and approximate hydrogen content are also included on this figure. A growing trend can be observed for both embrittlement indexes with increasing steel hardness. This trend is consistent with the change appreciated in the fracture micromechanisms. MVC was the only failure micromechanism in the grade with the lowest hardness, while $\mathrm{MVC}+\mathrm{PRHIC}$ and MVC+PRHIC+IG were the operative failure micromechanisms in the case of the grades with intermediate hardness and the highest hardness, respectively. Now, it should be recalled that hydrogen embrittlement is controlled by local stress and local hydrogen concentration, and only occurs when the critical combination of both factors is satisfied in some specific microstructure units.

The MVC micromechanism was always the only failure mechanism observed in the uncharged specimens of all grades, while the presence of hydrogen in the pre-charged specimens of the steel grade with the lowest hardness, 42CrMo4_700, although maintaining the same operative micromechanism, gave rise to larger and shallower dimples (see Fig. 9 a), as already stated in [20]. According to the hydrogen-enhanced localized plasticity mechanism (HELP) [7, 9, 20, 21], the presence of solute or internal hydrogen increases the mobility of dislocations enabling them to move under lower stresses, providing plasticity localization on the notch region, where shallow dimples are produced and grow, given rise to very large voids, as the ones shown in Fig. 9 (a). When this was the only operative micromechanism (i.e. in the steel grade with the lowest hardness, which is also the one with the lowest hydrogen content, 1.2-0.7 ppm), the effect of hydrogen was nearly negligible, as the embrittlement indexes were quite low (non-significant for the strength and around $20 \%$ for the reduction of area).

In all the other grades, ductile features (MVC) are observed in the center of the specimen and brittle areas at the notch tip region $[22,23]$ due to the stress profiles described in Figs. 10, 11, 12 (see also Figs. 9 c, 9 e). Failure initiates at the plastic zone at the tip of the notch, where normal and hydrostatic stresses are larger and hydrogen accumulates driven by the high hydrostatic stress. By the contrary, in the centre of the specimen and due to the relatively low level of the local hydrostatic stress, hydrogen difussed away and failure always took place in a ductile manner (MVC).

In the case of the grades with intermediate hardness, the existence of PRHIC was detected in the region just ahead of the notch. As was observed in Fig. 12, hydrostatic stress reaches its peak value at a depth between $80 \mu \mathrm{m}$ and $150 \mu \mathrm{m}$ for the different grades. This region suffer the greatest embrittlement, as it was shown in Figs. 9 c) and e), where quasi-brittle mechanisms were observed at a narrow peripheral region, whose extension increased from 100 to $250 \mu \mathrm{m}$, as tempering temperature reduces from 650 to $600^{\circ} \mathrm{C}$. PRHIC was the failure micromechanism observed in this region. It is related to hydrogen accumulation in martensite block and packet interphases, giving rise to local micro-plastic tearing on a very fine scale. It is seen that PRHIC requires a greater accumulation of hydrogen in the notch region and, in this case, it was due to the existence of higher hydrogen content and higher local hydrostatic stresses. The embrittlement produced by PRHIC (failure initiates just ahead of the notch under this mechanism and MVC takes place only afterwards in the centre of the specimen) was evident in Fig. 13, when the measured embrittlement indexes of the two grades with the lowest hardness are compared. Moreover, the area of the failure surface where PRHIC was detected was larger in the grades with the highest hardness (Fig. 9), the corresponding embrittlement indexes increase accordingly. 
Finally, intergranular fracture was observed in the steel grades with the highest hardness, also giving rise to the highest embrittlement indexes. The notched specimens of these steels also had higher hydrogen content and were subject to higher local hydrostatic stress. The accumulation of hydrogen ahead of the notch front was the highest, giving rise to decohesion of the prior austenitic grain boundaries (hydrogenenhanced decohesion mechanism, HEDE). In these cases, intergranular fracture is the first failure micromechanism to take place and gives rise to a substantial embrittlement, especially in the case of the grade with the highest hardness.

Recent studies have shown that intense slip bands (deformation bands) are present beneath intergranular and quasi-cleavage fracture surfaces as a consequence of the enhancement of dislocation activity by the hydrogen-enhanced localized plasticity (HELP) mechanism [7, 24, 25]. The reduction of notch area measured in all our tests, except for the steel grade with the highest strength under the lowest displacement rate, is the best evidence of the existence of plasticity in the process region before failure (see Table 5). Hydrogen redistribution occurs among the traps in front of the notch in the process region submitted to high hydrostatic stress, causing hydrogen accumulation on internal boundaries due to hydrogen transport by dislocations, until a crack initiates and propagates by interface decohesion driven by the action of the local stress [7, 8, 26]. Nagao et al. [25] explain this mechanism as a concerted action of the HELP and HEDE mechanisms whose operation is assisted by the hydrogen deposited on the internal boundaries and called it hydrogen-enhanced-plasticity mediated decohesion.

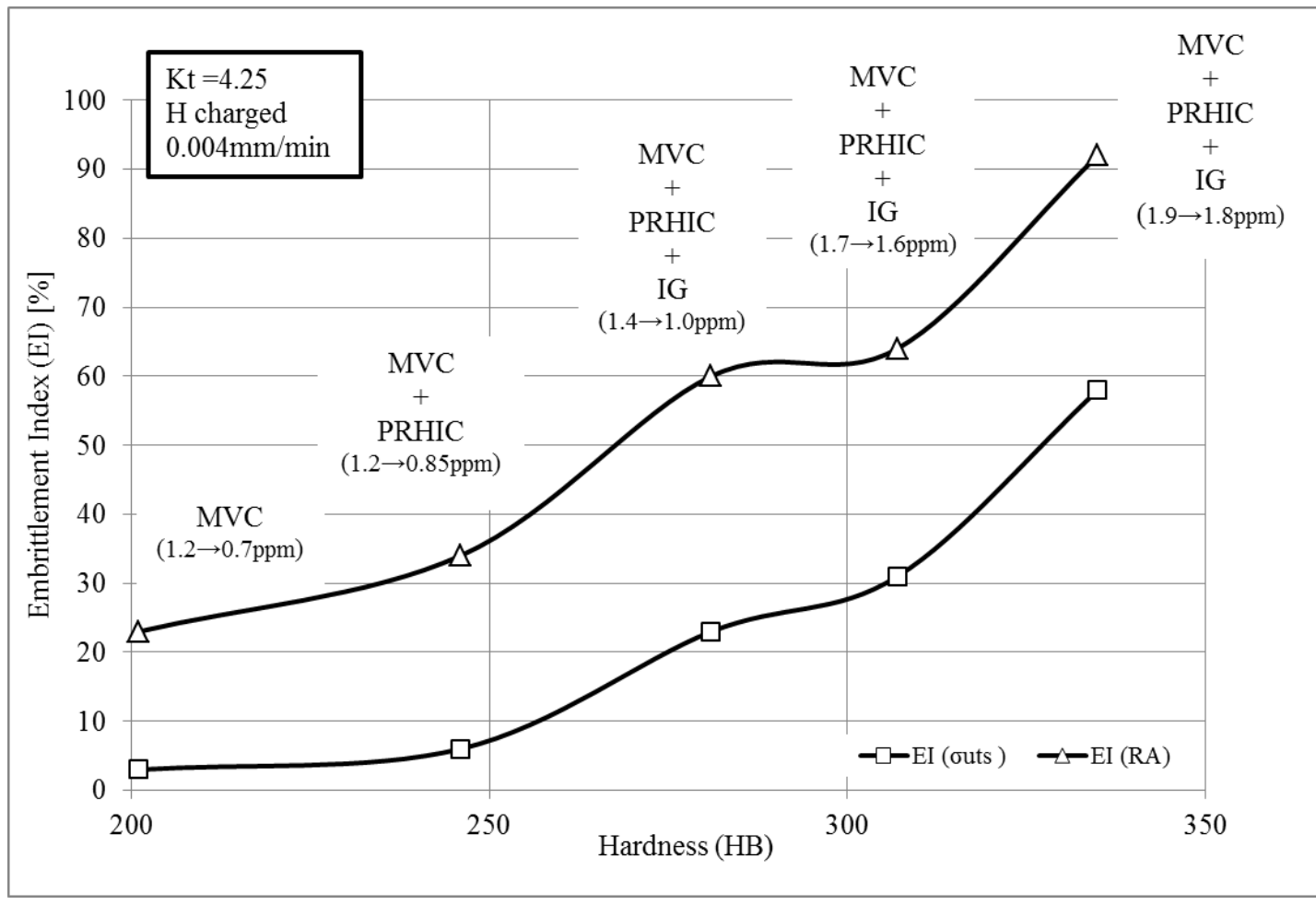

Fig. 13. Embrittlement indexes for notched tensile strength and reduction in area corresponding to each of the studied $42 \mathrm{CrMo} 4$ grades. Operative fracture micromechanisms and hydrogen content are included. Displacement rate: $0.004 \mathrm{~mm} / \mathrm{min}$.

Regarding the effects of microstructure on the hydrogen embrittlement of quenched and tempered steels, it should be recalled that after low temperature tempering, relaxation of the quenched microstructure is still quite low and an abundance of structural defects with a large capacity to accumulate hydrogen atoms can be found at the prior austenitic grain boundaries. In this situation, when internal hydrogen is present, hydrogen accumulates preferentially at the prior austenitic grain boundaries existing in the notch front region submitted to high hydrostatic stress, giving rise to decohesion of these interphases when a high enough local stress is applied. As a result, intergranular fracture takes place under a much lower tensile 
stress than the failure stress of the same specimen without hydrogen. As the tempering temperature increases, the steel microstructure relaxes, the distortion of the austenitic grain boundaries progressively diminish and their capacity to accumulate hydrogen decreases at the same time. In this case, the interphases between martensite blocks and packets are the sites where hydrogen accumulation is more abundant, fracture micromechanism changes from IG to PRHIC and embrittlement indexes decrease. It should also be noted that the surface per unit volume of all these interphases is much greater than those related to the austenitic grain boundaries. As the yield strength of the steels quenched and tempered at higher temperatures decreases, local normal stress and hydrostatic stress in the process zone diminish, contributing also to improve the embrittlement indexes. Only when the tempering temperature is high enough, microstructure relaxation reach a level at which hydrogen accumulation in all the aforementioned features is low and not sufficient to produce decohesion of any these interphases. In this situation, hydrogen distributes more uniformly in the notch front region and, due to HELP, dislocations move easily, plastic deformation in this region increases, the fracture micromechanism now becomes ductile (nucleation, growth and coalescence of microcavities, MVC), and failure takes place under only a slightly lower level of stress than in the absence of hydrogen. All these features are shown in Fig. 14.

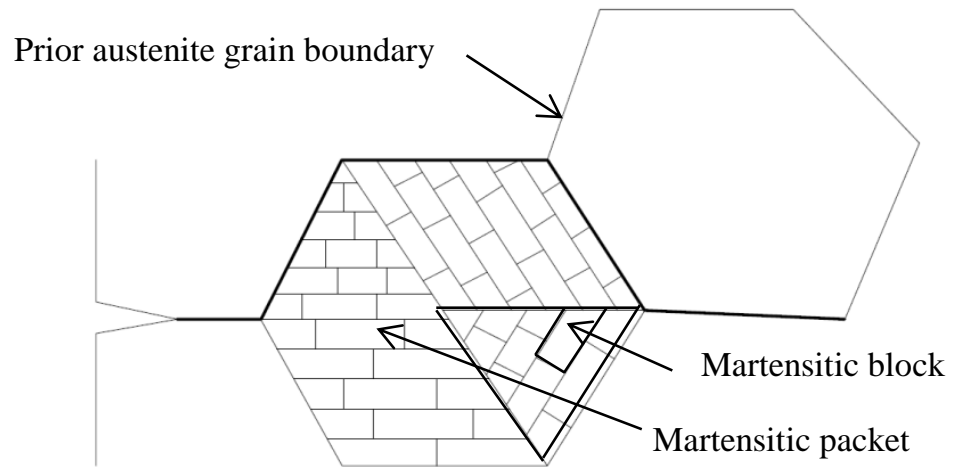

\section{LOW TEMPERING TEMPERATURE}

High hardness and yield strength Strongly distorted interphases

- High local hydrogen content

- High local stresses

(a) Low tempering temperature, $I G$

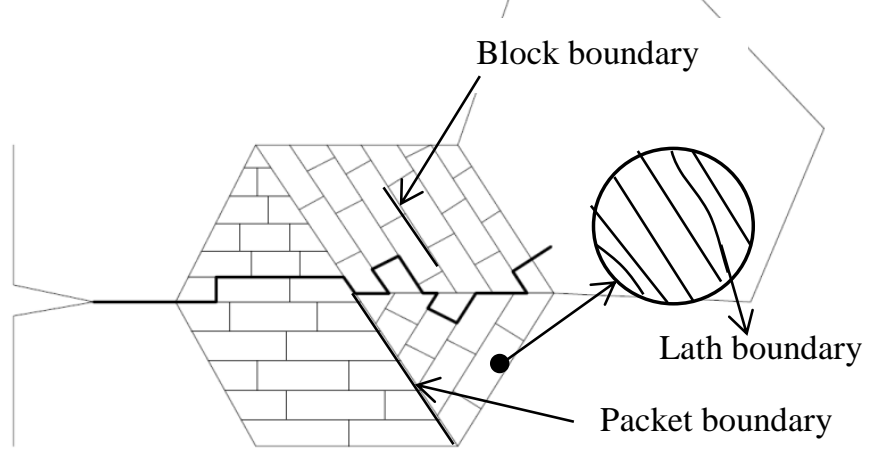

MEDIUM TEMPERING TEMPERATURE

Medium hardness and yield strength

Distorted interphases

- Medium local hydrogen content

- Medium local stresses

(b) Intermediate tempering temperature, PRHIC

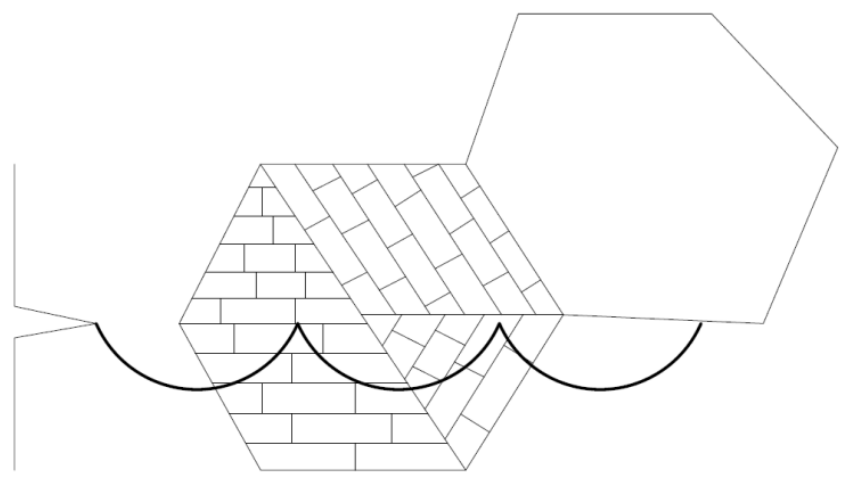

HIGH TEMPERING TEMPERATURE

Low hardness and yield strength

Relaxed interphases

- Low local hydrogen content

- $\quad$ Low local stresses

(c) High tempering temperature, $M V C$ 
Fig.14. Failure micromechanisms ahead of the notch in hydrogen charged quenched and tempered steels under similar stress concentrator and hydrogenated medium (a) IG, (b)

PRHIC and (c) MVC.

\section{CONCLUSIONS}

. The initial hydrogen content, measured in samples pre-charged under high temperature hydrogen pressure, increased with decreasing steel tempering temperature due to the fact that hydrogen microstructural trapping is greater in distorted, high energy martensitic microstructures. Moreover, the content of hydrogen strongly trapped is even more dependent on the steel microstructure, decreasing with increasing tempering temperature, mainly due to stress relaxation and the reduction in dislocation density and interphases (martensite laths, blocks and packets).

. The tensile properties measured on smooth specimens pre-charged in gaseous hydrogen remained practically unaffected, irrespective of the applied displacement rate.

. The embrittlement indexes (strength and reduction in area) obtained using hydrogen pre-charged notch tensile specimens increase with steel hardness. This trend is consistent with the change appreciated in the fracture micromechanisms. MVC was the only failure micromechanism in the grade with the lowest hardness, while MVC+PRHIC and MVC+PRHIC+IG were the operative failure micromechanisms in the case of the grades with intermediate hardness and the highest hardness, respectively.

. The use of lower displacement rates always gave rise to higher embrittlement indexes, as the accumulation of hydrogen atoms in the most stressed area of the notched specimen increases due to the availability of longer diffusion times.

. Finally, these results are explained by the magnitude of the hydrostatic and normal stresses existing in the vicinity of the notch (which are related with the steel yield strength), and by differences in local hydrogen accumulation within the different microstructural features.

\section{AKNOWLEDGEMENTS}

The authors would like to thank the Spanish Ministry of Economy and Competitiveness for the support received to carry out research project MAT2014-58738-C3 (SAFEHIDROSTEEL).

\section{REFERENCES}

[1] J. Yamabe, T. Awane, and S. Matsuoka, "ScienceDirect Investigation of hydrogen transport behavior of various low-alloy steels with high-pressure hydrogen gas", Int. J. Hydrogen Energy, vol. 40, no. 34, pp. 11075-11086, 2015.

[2] J. H. Chuang, L. W. Tsay, and C. Chen, "Crack growth behaviour of heat-treated 4140 steel in air and gaseous hydrogen", Int. J. Fatigue, vol. 20, no. 7, pp. 531-536, 1998.

[3] L. Briottet, R. Batisse, G. de Dinechin, P. Langlois, and L. Thiers, "Recommendations on X80 steel for the design of hydrogen gas transmission pipelines", Int. J. Hydrogen Energy, vol. 37, no. 11, pp. 9423-9430, 2012.

[4] M. Wang, E. Akiyama, and K. Tsuzaki, "Effect of hydrogen on the fracture behavior of high strength steel during slow strain rate test", Corros. Sci., vol. 49, no. 11, pp. 4081-4097, 2007. 
[5] E. Akiyama, M. Wang, S. Li, Z. Zhang, Y. Kimura, N. Uno, K. Tsuzaki, "Studies of evaluation of hydrogen embrittlement property of high-strength steels with consideration of the effect of atmospheric corrosión", Metallurgical and Materials Transactions, vol. 44A, pp. 1290-1300, 2013.

[6] D.K. Singh, R.K.S. Raman, S.K. Maiti, T.K. Bhandakkar, S. Pal, "Investigation of role of alloy microstructure of AISI 4340 steel using circumferentially notched cylindrical specimens", Materials Science \& Engineering A, vol. 698, pp. 191-197, 2017.

[7] A. Nagao, C.D. Smith, M. Dadfarnia, P. Sofronis, I.M. Robertson, "Interpretation of hydrogeninduced fracture surface morphologies for lath martensitic steel", Procedia Materials Science, vol. 3, pp. 1700-1705, 2014.

[8] I. M. Robertson, P. Sofronis, A. Nagao, M.L. Martin, S. Wang, D.W. Gross. K.E. Nygren, "Hydrogen embrittlement understood", Metallurgical and Materials Transactions, vol. 46B, pp. 1085-1103, 2015.

[9] R.P. Gangloff, B.P. Somerday Edit., "Gaseous hydrogen embrittlement of materials in energy technologies", Woodhead Publishing Limited, Cambridge, UK, 2012.

[10] A. Oudriss, A. Fleurentin, G. Courlit, E. Conforto, C. Berziou, C. Rebéré, S. Cohendoz, J.M. Sobrino, J. Creus, X. Feugas, "Consequence of the diffusive hydrogen contents on tensile properties of martensitic steel during the desorption at room temperature", Materials Science \& Engineering A, vol. 598, pp. 420-428, 2014.

[11] E.J. Song, S.W. Baek, S.H. Nahm, U.B. Baek, "Notched-tensile properties under high-pressure gaseous hydrogen: Comparison of pipeline Steel X70 and austenitic type 304L 316L steels", Int. J. Hydrogen Energy, vol. 42, pp. 8075-8082, 2017.

[12] ASTM G146, "Evaluation of disbonding of bimetallic stainless alloy/steel plate for use in highpressure, high temperature refinery hydrogen service", Annual Book of ASTM Standards, Vol. $03.02,2013$.

[13] H. Neuber, F. A. Raven, and J. S. Brock, Theory of Notch Stresses:principles for exact stress calculation. Berlin: Julius Springer, 1937.

[14] S. Takagi, S. Terasaki, K. Tsuzaki, T. Inoue, and F. Minami, "Application of Local Approach to Hydrogen Embrittlement Fracture Evaluation of High Strength Steels", Materials Science Forum vol. 539-543, pp. 2155-2161, 2007.

[15] George Krauss, Steels: Processing, Structure and Performance. 2005.

[16] Y. Takeda and C. J. McMahon Jr., "Strain controlled vs. qtress controlled hydrogen induced fracture in a quenched and qempered steel," Metall. Mater. Trans. A, vol. 12A, pp. 1255-1266, 1981.

[17] M. Wang, E. Akiyama, and K. Tsuzaki, "Effect of hydrogen and stress concentration on the notch tensile strength of AISI 4135 steel", Materials Science \& Engineering A, vol. 398, pp. 37-46, 2005.

[18] K. A. Nibur, B. P. Somerday, C. S. A. N. Marchi, J. W. Foulk, M. Dadfarnia, and P. Sofronis, "The Relationship Between Crack-Tip Strain and Subcritical Cracking Thresholds for Steels in HighPressure Hydrogen Gas," Metall. Mater. Trans. A, vol. 44A, pp. 248-269, 2013.

[19] G. M. Pressouyre, "Trap theory of Hydrogen embrittlement", Acta Metall., vol. 28, no. 7, pp. 895911,1980

[20] T. Matsuo, N. Homma, S. Matsuoka, Y. Murakami, "Effect of hydrogen and prestrain on tensile properties of carbon steel SGP (0.078C-0.012Si-0.35Mn, mass\%) for 0.1 MPa hydrogen pipelines", Transactions of the JSME A, vol. 74 (744), pp. 1165-1173, 2008.

[21] Y. Murakami and S. Matsuoka, "Effect of hydrogen on fatigue crack growth of metals," Eng. Fract. Mech., vol. 77, no. 11, pp. 1926-1940, 2010. 
[22] Y.W. Sun, J.Z. Chen, Investigation into hydrogen diffusion and susceptibility of hydrogen embrittlement of high strength 0Cr16Ni5Mo steel, Journal of Iron and Steel Research International, vol. 22, no. 10, pp. 961-968 2015.

[23] T. An, S. Zheng, H. Peng, X. Weng, L. chen, L. Zhang, "Synergistic action of hydrogen and stress concentration on the fatigue properties of X80 pipeline steel”, Materials Science \&Engineering A, vol.700, pp. 321-330, 2017.

[24] P. Novak, R. Yuan, B.P. Somerday, P. Sofronis, R.O. Ritchie, "A statistical physical-based, micomechanical model of hydrogen-induced intergranular fracture in steel", Journal of the Mechanics and Physics of Solids, vol. 58, pp. 206-226, 2010.

[25] A. Nagao, M. Dadfarnia, B.P. Somerday, P. Sofronis, R.O. Ritchie, "Hydrogen-enhanced-plasticity mediated decohesion for hydrogen-induced intergraular and quasi-cleavage fracture of lath martensitic steels", Journal of the Mechanics and Physics of Solids, vol. 112, pp 403-430, 2018.

[26] T. Michler, C. San Marchi, J. Naumann, S. Weber, M. Martin, "Hydrogen environment embrittlement of stable austenitic steels", International Journal of Hydrogen Energy, vol. 37, pp. 16231-16246, 2012 